\title{
MAXIMUM PRINCIPLES AND MONOTONICITY OF SOLUTIONS FOR FRACTIONAL P-EQUATIONS IN UNBOUNDED DOMAINS
}

\author{
ZHAO LIU ${ }^{\dagger}$, WENXIONG CHEN $* \ddagger$
}

ABStRACT. In this paper, we consider the following non-linear equations in unbounded domains $\Omega$ with exterior Dirichlet condition:

$$
\begin{cases}(-\Delta)_{p}^{s} u(x)=f(u(x)), & x \in \Omega, \\ u(x)>0, & x \in \Omega, \\ u(x) \leq 0, & x \in \mathbb{R}^{n} \backslash \Omega,\end{cases}
$$

where $(-\Delta)_{p}^{s}$ is the fractional p-Laplacian defined as

$$
(-\Delta)_{p}^{s} u(x)=C_{n, s, p} P . V . \int_{\mathbb{R}^{n}} \frac{|u(x)-u(y)|^{p-2}[u(x)-u(y)]}{|x-y|^{n+s p}} d y
$$

with $0<s<1$ and $p \geq 2$.

We first establish a maximum principle in unbounded domains involving the fractional p-Laplacian by estimating the singular integral in (0.1) along a sequence of approximate maximum points. Then, we obtain the asymptotic behavior of solutions far away from the boundary. Finally, we develop a sliding method for the fractional p-Laplacians and apply it to derive the monotonicity and uniqueness of solutions.

There have been similar results for the regular Laplacian 1 and for the fractional Laplacian [30, which are linear operators. Unfortunately, many approaches there no longer work for the fully non-linear fractional p-Laplacian here. To circumvent these difficulties, we introduce several new ideas, which enable us not only to deal with non-linear non-local equations, but also to remarkably weaken the conditions on $f(\cdot)$ and on the domain $\Omega$.

We believe that the new methods developed in our paper can be widely applied to many problems in unbounded domains involving non-linear non-local operators.

Keywords: Fractional p-Laplacians, maximum principles, unbounded domains, sliding methods, asymptotic behavior, monotonicity of solutions, uniqueness.

2010 MSC Primary: 35R11, 35J91; Secondary: 35B06, 35B65.

\section{INTRODUCTION}

In this paper, we investigate qualitative properties of solutions for the nonlinear problem

$$
\begin{cases}(-\Delta)_{p}^{s} u(x)=f(u(x)), & x \in \Omega, \\ u(x)>0, & x \in \Omega, \\ u(x) \leq 0, & x \in \mathbb{R}^{n} \backslash \Omega,\end{cases}
$$

where $\Omega$ is the region above the graph of a continuous function $\varphi: \mathbb{R}^{n-1} \rightarrow \mathbb{R}$, i.e.

$$
\Omega:=\left\{x=\left(x^{\prime}, x_{n}\right) \in \mathbb{R}^{n} \mid x_{n}>\varphi\left(x^{\prime}\right)\right\} \text { with } x^{\prime}=\left(x_{1}, x_{2}, \cdots, x_{n-1}\right) \in \mathbb{R}^{n-1} .
$$

* Corresponding author: Wenxiong Chen at wchen@yu.edu. Zhao Liu is supported by the NNSF of China (No. 11801237). 
$(-\Delta)_{p}^{s}$ is the fractional p-Laplacian defined as

$$
\begin{aligned}
(-\Delta)_{p}^{s} u(x) & =C_{n, s, p} P . V . \int_{\mathbb{R}^{n}} \frac{|u(x)-u(y)|^{p-2}[u(x)-u(y)]}{|x-y|^{n+s p}} d y \\
& =C_{n, s, p} \lim _{\varepsilon \rightarrow 0} \int_{\mathbb{R}^{n} \backslash B_{\varepsilon}(x)} \frac{|u(x)-u(y)|^{p-2}[u(x)-u(y)]}{|x-y|^{n+s p}} d y,
\end{aligned}
$$

where P.V. stands for the Cauchy principal value, and throughout this paper, we assume that $s \in(0,1)$ and $p \geq 2$.

In order the integral to make sense, we require that

$$
u \in C_{l o c}^{1,1} \cap \mathcal{L}_{s p}
$$

with

$$
\mathcal{L}_{s p}:=\left\{u \in L_{l o c}^{p-1} \mid \int_{\mathbb{R}^{n}} \frac{|u(x)|^{p-1}}{1+|x|^{n+s p}} d x<\infty\right\} .
$$

In the special case when $p=2,(-\Delta)_{p}^{s}$ becomes the well-known fractional Laplacian $(-\Delta)^{s}$. And one can show that, as $s \rightarrow 1$, the fractional p-Laplacian converges to the regular $\mathrm{p}$ Laplacian:

$$
(-\Delta)_{p}^{s} u(x) \rightarrow-\Delta_{p} u(x):=-\operatorname{div}\left(|\nabla u(x)|^{p-2} \nabla u(x)\right)
$$

The non-local nature of these operators make them difficult to study. To circumvent this, Caffarelli and Silvestre [9] introduced the extension method which turns the non-local problem involving the fractional Laplacian into a local one in higher dimensions. This method has been applied successfully to study equations involving the fractional Laplacian, and a series of fruitful results have been obtained (see [6, 23] and the references therein). One can also use the integral equations method, such as the method of moving planes in integral forms and regularity lifting to investigate equations involving the fractional Laplacian by first showing that they are equivalent to the corresponding integral equations (see [13, 20, 21]).

However, so far as we know, besides the fractional Laplacian, there has not been any extension methods that works for other non-local operators, such as the uniformly elliptic non-local operators and fully non-linear non-local operators (see [10] for the introductions of these operators) including the fractional p-Laplacian. In [18], Chen, Li and Li introduced the direct method of moving planes for the fractional Laplacian which has been applied to obtain symmetry, monotonicity, and non-existence of solutions for various semi-linear equations involving the fractional Laplacian. In [17], Chen, Li and Li refined this direct approach, so that it can be applied to fully nonlinear nonlocal problem in the case the operator is nondegenerate in certain sense. In order to investigate the degenerate fractional p-Laplacian, Chen and $\mathrm{Li}$ [16] introduced some new ideas, among which a significant one is a variant of the Hopf Lemma, the key boundary estimate, which plays the role of the narrow region principle in the second step of the method of moving planes. For more applications about this direct method for various non-local problems, please see [19, 22] and the references therein.

It is well-known that maximum principles play fundamental roles in the study of elliptic partial differential equations, it is also a powerful tool in carrying out the method of moving planes to derive symmetry, monotonicity, and non-existence of solutions. Recently, due to their broad applications to various branches of sciences, a lot of attention has been turned to the non-linear equations involving fractional Laplacians and other non-local operators, including the fully non-linear non-local fractional p-Lapcians. In order to further investigate 
these non-local equations, here we establish a fractional p-Laplacian version of the maximum principle in unbounded domains without assuming any asymptotic behavior of the solutions near infinity, which can be applied to establish qualitative properties, such as symmetry and monotonicity for solutions of fractional p-Laplacian equations.

Our first result is the following.

Theorem 1. Let $D$ be a open set in $\mathbb{R}^{n}$, possibly unbounded and disconnected. Assume that $\bar{D}$ is disjoint from the closure of an infinite open domain $\Sigma$ satisfying

$$
\underline{\lim }_{j \rightarrow \infty} \frac{\left|\left(B_{2^{j+1} r}(x) \backslash B_{2^{j} r}(x)\right) \cap \Sigma\right|}{\left|B_{2^{j+1} r}(x) \backslash B_{2^{j} r}(x)\right|} \geq c_{0}, \quad \forall x \in \mathbb{R}^{n},
$$

for some $c_{0}>0$ and $r>0$. Suppose $u(x)$ is in $C_{l o c}^{1,1} \cap \mathcal{L}_{s p}$, bounded from above, and satisfies

$$
\begin{cases}(-\Delta)_{p}^{s} u(x)+c(x) u(x) \leq 0, & x \in D, \\ u(x) \leq 0, & x \in \mathbb{R}^{n} \backslash D,\end{cases}
$$

for some nonnegative function $c(x)$.

Then $u(x) \leq 0$ in $D$.

A similar maximum principle in unbounded domains in the classical case involving the regular Laplacian (when $s=1, p=2$ ) was obtained by Berestycki, Caffarelli and Nirenberg [1]. Birindelli and Prajapat [5] extended the maximum principle to the Heisenberg group. For the fractional Laplacian (when $s \in(0,1), p=2$ ), Dipierro, Soave and Valdinoci [30] proved the same results based on growth lemmas established by De Giorgi [28] and Silvestre [46] respectively.

In both of the above articles [1,30], the authors assumed that the complement of $\bar{D}$ contains an infinite open cone $\Sigma$. One may call this an exterior cone condition. It is easy to check that the infinite open cone satisfies (1.2) in our Theorem 1. Actually, one can see that our condition (1.2) is much weaker than the exterior cone condition. There are many domains $\bar{D}$ whose complement do not contain an infinite cone. To illustrate this, we list the following two simple examples of such domains.

(a) $D=\left\{x \in \mathbb{R}^{n} \mid 2 i<x_{n}<2 i+1, i=0, \pm 1, \pm 2, \cdots\right\}$.

(b) $D=\left\{x \in \mathbb{R}^{n}|2 i<| x \mid<2 i+1, i=0,1,2, \cdots\right\}$.

Obviously, none of the above two domains $D$ satisfy the exterior cone condition. Since our Theorem 1 includes the case when $p=2$, it improves the result in [30] by weakening the condition on the domains.

We would like to mention that the operators $-\Delta$ and $(-\Delta)^{s}$ considered in [1] and [30] respectively are linear ones, while the fractional p-Laplacian $(-\Delta)_{p}^{s}$ in this paper is fully non-linear. Hence the methods in [1] and [30] can no longer be applied here. To deal with such non-local non-linear operators, we introduce new ideas.

Usually, to prove a maximum principle on bounded domains, or on unbounded domains assuming that the solutions vanishes near infinity, one derives contradictions at a maximum point. However, on unbounded domains without imposing any asymptotic conditions on the solution $u$, the maximum value of $u$ may not be attained, and a maximizing sequence 
may tend to infinity. To circumvent this difficulty, we estimate the singular integral defining $(-\Delta)_{p}^{s} u$ along a sequence of approximate maximum points to derive a contradiction if, in Theorem 1, $\sup _{D} u(x)>0$. It turns out that this approach is quite simple, and it also applies to the case of fractional Laplacian (when $p=2$ ). We believe that this method will become a very useful tool to investigate many other non-linear equations involving general non-linear non-local operators.

The moving plane method and the sliding method are techniques that have been used in recent years to establish qualitative properties of solutions of non-linear elliptic equations such as symmetry, monotonicity, and non-existence. In fact, the method of moving planes was initially invented by Alexanderoff in the early 1950s. Later, it was further developed by Serrin [47], Gidas, Ni and Nirenberg [33, 34], Caffarelli, Gidas and Spruck [8], Chen and Li [14, Li and Zhu [38], Chang and Yang [11, Lin [36] and many others. For more literatures about the method of moving planes, please refer to [13, 15, 22, 24, 25, 26, 27, 32, 37, 41, 42, 43, 44, 48, 49] and the references therein. The sliding method was introduced by Berestycki, Caffarelli and Nirenberg [1, 2, 4], which is slightly different from the method of moving plane, it is used to compare the solution with its translation rather than its reflection. The sliding method was also successful in obtaining symmetry and monotonicity of solutions for many kind of domains (see [2, 3] ).

We consider the following fractional p-Laplacian equation

$$
\begin{cases}(-\Delta)_{p}^{s} u(x)=f(u(x)), & x \in \Omega, \\ u(x)>0, & x \in \Omega .\end{cases}
$$

As preparations to carry out the sliding method along $x_{n}$-direction, we obtain the following two theorems, which may also be applied to other situations.

Theorem 2. Let $u \in C_{l o c}^{1,1} \cap \mathcal{L}_{s p}$ be a bounded solution of (1.4). Assume that $f$ is continuous and satisfies

(a) There exists $\mu>0$ such that $f(t)>0$ on $(0, \mu)$, and $f(t) \leq 0$ for $t \geq \mu$.

Suppose that $u(x)<\mu, x \in \mathbb{R}^{n} \backslash \Omega$, then

$$
u(x)<\mu \text { for all } x \in \Omega \text {. }
$$

Theorem 3. Let $u \in C_{l o c}^{1,1} \cap \mathcal{L}_{s p}$ be a bounded solution of (1.4) with

$$
0 \leq u(x)<\mu, \quad x \in \mathbb{R}^{n} \backslash \Omega .
$$

Assume that $f$ is continuous, satisfies condition (a) and for some $0<t_{0}<t_{1}<\mu$,

(b) $f(t) \geq \delta_{0} t$ on $\left[0, t_{0}\right]$ for some $\delta_{0}>0$, and

(c) $f(t)$ is nonincreasing on $\left(t_{1}, \mu\right)$.

Then $u(x) \rightarrow \mu$ uniformly in $\Omega$ as $\operatorname{dist}(x, \partial \Omega) \rightarrow \infty$.

Let us point out that Theorem 2 and Theorem 3 are closely related to the well-known $D e$ Giorgi conjecture:

Conjecture 1. (De Giorgi [29]). If $u$ is a solution of

$$
-\Delta u=u-u^{3}
$$


such that

$$
|u| \leq 1 \text { in } \mathbb{R}^{n}, \lim _{x_{n} \rightarrow \pm \infty} u\left(x^{\prime}, x_{n}\right)= \pm 1 \text { for all } x^{\prime} \in \mathbb{R}^{n-1}
$$

and

$$
\frac{\partial u}{\partial x_{n}}>0
$$

Then there exists a vector $a \in \mathbb{R}^{n-1}$ and a function $u_{1}: \mathbb{R} \rightarrow \mathbb{R}$ such that

$$
u\left(x^{\prime}, x_{n}\right)=u_{1}\left(a \cdot x^{\prime}+x_{n}\right) \text { in } \mathbb{R}^{n} .
$$

If we replace $-\Delta$ by $(-\Delta)_{p}^{s}$, take $\mu=1$, and $f(u)=u-u^{3}$ as in the De Giorgi's conjecture, then conditions (a)-(c) in Theorem 3 are satisfied. Hence we derive that

$$
u<1 \text { in } \Omega \text { and } u(x) \rightarrow 1 \text { uniformly in } \Omega \text { as } \operatorname{dist}(x, \partial \Omega) \rightarrow \infty .
$$

Therefore, we can replace condition (1.5) by

$$
u>0 \text { for } x_{n}>M\left(\text { take } \varphi\left(x^{\prime}\right) \equiv M\right) .
$$

Based on the above two theorems, we will apply the sliding method to obtain the monotonicity of solutions for the following problem.

$$
\begin{cases}(-\Delta)_{p}^{s} u(x)=f(u(x)), & x \in \Omega, \\ u(x)>0, & x \in \Omega, \\ u(x)=0, & x \in \mathbb{R}^{n} \backslash \Omega,\end{cases}
$$

where $\Omega$ satisfies the uniform two-sided ball condition (the exterior and interior ball conditions).

We prove

Theorem 4. Let $u \in C_{\text {loc }}^{1,1} \cap \mathcal{L}_{\text {sp }}$ be a bounded solution of (1.7). Assume that $f$ is a continuous function and satisfies conditions (a)-(c) for some $0<t_{0}<t_{1}<\mu$.

Then $u$ is strictly monotone increasing in $x_{n}$.

Furthermore, the bounded solution of (1.7) is unique.

Remark 1. Our results in this paper adapt to the case of the fractional Laplacian where $p=2$. As prototype in Theorem 4, we may take $f(u)=u-u^{3}$ or $f(u)=u-u^{2}$. Then equation (1.7) is the well-known fractional Allen-Cahn equation or the fractional Fisher-Kolmogorov equation, which have been widely studied by many authors (please see [12, 31, 45] and the references therein).

Remark 2. Theorem 4 was proved by Berestycki, Caffarelli and Nirenberg [1] for $s=1$ and $p=2$, and Dipierro, Soave and Valdinoci [30] for $s \in(0,1)$ and $p=2$ respectively. They all assumed $f(\cdot)$ to be globally Lipschitz continuous. In this paper we only require $f(\cdot)$ to be continuous, which is weaker than the condition in the classical results established by Berestycki, Caffarelli and Nirenberg [1] and Dipierro, Soave and Valdinoci [30]. This is mainly because we employ a new and different idea here. 
To illustrate the major differences between the traditional approach and our approach, let

$$
x=\left(x^{\prime}, x_{n}\right), u_{\tau}(x)=u\left(x^{\prime}, x_{n}+\tau\right), \text { and } w_{\tau}(x)=u(x)-u_{\tau}(x) .
$$

To obtain the result in Theorem 4, one first needs to show that

$$
w_{\tau}(x) \leq 0, \quad \forall \tau>0, x \in \Omega .
$$

This is achieved via a contradiction argument. Suppose $\sup w_{\tau}=A>0$, then there exists a sequence $\left\{x^{k}\right\}$, such that

$$
w_{\tau}\left(x^{k}\right) \rightarrow A, \text { as } k \rightarrow \infty .
$$

Making the translation $w_{\tau}^{k}(x)=w_{\tau}\left(x+x^{k}\right)$, in the linear operator case as in [1] and [30], they obtained

$$
(-\Delta)^{s} w_{\tau}^{k}(x)=c_{k}(x) w_{\tau}^{k}(x) .
$$

Here $c_{k}(x)$ are uniformly bounded due to the global Lipshitz continuity assumption on $f$. Based on this, they were able to show that

$$
w_{\tau}^{k}(x) \rightarrow w_{\tau}^{\infty}(x) \text { and }(-\Delta)^{s} w_{\tau}^{k}(x) \rightarrow(-\Delta)^{s} w_{\tau}^{\infty}(x)
$$

and therefore

$$
(-\Delta)^{s} w_{\tau}^{\infty}(x)=c_{\infty}(x) w_{\tau}^{\infty}(x) \text { with } w_{\tau}^{\infty}(0)=A>0 .
$$

Then, they were able to derive a contradiction based on the properties of the solutions of the above equation.

In our nonlinear operator case, the first difficulty is

$$
(-\Delta)_{p}^{s} u(x)-(-\Delta)_{p}^{s} u_{\tau}(x) \neq(-\Delta)_{p}^{s} w_{\tau}(x) .
$$

Hence the simple maximum principle such as Theorem 1 can not be applied directly. We will modify it in the proof of Theorem 4 .

The second difficulty is more subtle. So far, there have been very few regularity results on the solutions for fractional p-equations, the best we know is that the solutions $u$ are uniformly Hölder continuous if both $u$ and $f(u)$ are bounded. These are far from sufficient to guarantee the convergence of

$$
(-\Delta)_{p}^{s} u^{k}(x)-(-\Delta)_{p}^{s} u_{\tau}^{k}(x),
$$

which requires $\left\{u^{k}\right\}$ to be uniformly $C^{1,1}$.

To circumvent this difficulty, instead of estimating along a sequence of equations in the whole domain $\Omega$, we estimate the singular integrals defining $(-\Delta)_{p}^{s} u(x)-(-\Delta)_{p}^{s} u_{\tau}(x)$ only along a sequence of points, the approximate maximum points $x^{k}$. This new idea not only enable us to deal with the situation where the lack of the regularity result is known, but also enable us to weaken the condition on the nonlinearity $f(u)$.

Finally, we consider a special case where $\Omega$ is an upper half space:

$$
\begin{cases}(-\Delta)_{p}^{s} u(x)=f(u(x)), & x \in \mathbb{R}_{+}^{n}, \\ u(x)>0, & x \in \mathbb{R}_{+}^{n}, \\ u(x)=0, & x \in \mathbb{R}^{n} \backslash \mathbb{R}_{+}^{n} .\end{cases}
$$

For this particular domain, we are able to use the sliding method in any direction to obtain a stronger result. 
Theorem 5. Suppose that $u \in C_{l o c}^{1,1} \cap \mathcal{L}_{s p}$ be a bounded solution of (1.8). Assume that $f$ is continuous and satisfies conditions (a)-(c) for some $0<t_{0}<t_{1}<\mu$.

Then $u$ is strictly monotone increasing in $x_{n}$, and moreover it depends on $x_{n}$ only.

Furthermore the bounded solution of (1.8) is unique.

The rest of our paper is organized as follows. In section 2, we prove the maximum principles in unbounded domains and hence establish Theorem 1. Based on the maximum principles, we obtain Theorem [2. In section 3, we carry out our proof of Theorem 3 by using a sliding method on ball regions. In section 4 , we prove the monotonicity and uniqueness by estimating the singular integrals along the approximate maximum points in the process of sliding and thus obtain Theorem 4. Section 5 is devoted to proving Theorem 5 .

In the following, we will use $C$ to denote a general positive constant that may depend on $n, s$ and $p$, and whose value may differ from line to line.

\section{The proof of Theorem 1 and Theorem 2}

In this section, we establish the following maximum principles in unbounded domains.

Theorem 2.1. Let $D$ be a open set in $\mathbb{R}^{n}$, possibly unbounded and disconnected. Assume that $\bar{D}$ is disjoint from the closure of an infinite open domain $\Sigma$ satisfying

$$
\varliminf_{j \rightarrow \infty} \frac{\left|\left(B_{2^{j+1} r}(x) \backslash B_{2^{j} r}(x)\right) \cap \Sigma\right|}{\left|B_{2^{j+1} r}(x) \backslash B_{2^{j} r}(x)\right|} \geq c_{0}, \quad \forall x \in \mathbb{R}^{n},
$$

for some $c_{0}>0$ and $r>0$. Suppose $u(x)$ is in $C_{l o c}^{1,1} \cap \mathcal{L}_{s p}$, bounded from above, and satisfies

$$
\begin{cases}(-\Delta)_{p}^{s} u(x)+c(x) u(x) \leq 0, & x \in D, \\ u(x) \leq 0, & x \in \mathbb{R}^{n} \backslash D,\end{cases}
$$

for some nonnegative function $c(x)$.

Then $u(x) \leq 0$ in $D$.

Proof. Suppose on the contrary, there is some points $x$ such that $u(x)>0$ in $D$, then

$$
0<A:=\sup _{x \in \mathbb{R}^{n}} u(x)<\infty .
$$

There exists sequences $x^{k} \in D$ and $\gamma_{k} \rightarrow 1\left(\gamma_{k} \in(0,1)\right)$ as $k \rightarrow \infty$ such that

$$
u\left(x^{k}\right) \geq \gamma_{k} A \text {. }
$$

Let

$$
\Phi(x)= \begin{cases}c_{n} e^{\frac{1}{|x|^{2}-4}}, & |x|<2 \\ 0, & |x| \geq 2\end{cases}
$$

It is easy to check that $\Phi$ is radially decreasing from the origin, and is in $C_{0}^{\infty}\left(B_{2}(0)\right)$. Define

$$
\Phi_{r_{k}}(x):=\Phi\left(\frac{x-x^{k}}{r_{k}}\right)
$$


For any $x \in B_{2 r_{k}}\left(x^{k}\right) \backslash B_{r_{k}}\left(x^{k}\right)$, we can take $\varepsilon_{k}>0$ such that

$$
u\left(x^{k}\right)+\varepsilon_{k} \Phi_{r_{k}}\left(x^{k}\right) \geq A+\varepsilon_{k} \Phi_{r_{k}}\left(x^{k}+r_{k} e\right) \geq u(x)+\varepsilon_{k} \Phi_{r_{k}}(x) .
$$

where $e$ is any unit vector in $\mathbb{R}^{n}$.

Therefore, there exists $\bar{x}^{k} \in B_{r_{k}}\left(x^{k}\right)$ such that

$$
u\left(\bar{x}^{k}\right)+\varepsilon_{k} \Phi_{r_{k}}\left(\bar{x}^{k}\right)=\max _{x \in B_{2 r_{k}}\left(x^{k}\right)}\left[u(x)+\varepsilon_{k} \Phi_{r_{k}}(x)\right] .
$$

As a consequence,

$$
u\left(\bar{x}^{k}\right)+\varepsilon_{k} \Phi_{r_{k}}\left(\bar{x}^{k}\right) \geq u\left(x^{k}\right)+\varepsilon_{k} \Phi_{r_{k}}\left(x^{k}\right),
$$

which implies

$$
u\left(\bar{x}^{k}\right) \geq u\left(x^{k}\right)+\varepsilon_{k} \Phi_{r_{k}}\left(x^{k}\right)-\varepsilon_{k} \Phi_{r_{k}}\left(\bar{x}^{k}\right) \geq u\left(x^{k}\right) .
$$

It follows from (2.4) that

$$
u\left(\bar{x}^{k}\right) \geq \gamma_{k} A .
$$

From (2.7) and (2.8), we deduce that

$$
u\left(\bar{x}^{k}\right)+\varepsilon_{k} \Phi_{r_{k}}\left(\bar{x}^{k}\right) \geq A \geq u(x), \forall x \in \mathbb{R}^{n} .
$$

Hence $\bar{x}^{k}$ is a maximum of the function $u+\varepsilon_{k} \Phi_{r_{k}}$ in $\mathbb{R}^{n}$.

Let $G(t)=|t|^{p-2} t$, we calculate

$$
\begin{aligned}
(-\Delta)_{p}^{s} u\left(\bar{x}^{k}\right)+\frac{\varepsilon_{k}^{p-1}}{r_{k}^{s p}}\left[(-\Delta)_{p}^{s} \Phi\right]\left(\frac{\bar{x}^{k}-x^{k}}{r_{k}}\right) \\
=(-\Delta)_{p}^{s} u\left(\bar{x}^{k}\right)+(-\Delta)_{p}^{s}\left[\varepsilon_{k} \Phi_{r_{k}}\left(\bar{x}^{k}\right)\right] \\
=C_{n, s, p} P . V \cdot \int_{\mathbb{R}^{n}} \frac{G\left(u\left(\bar{x}^{k}\right)-u(y)\right)+G\left(\varepsilon_{k} \Phi_{r_{k}}\left(\bar{x}^{k}\right)-\varepsilon_{k} \Phi_{r_{k}}(y)\right)}{\left|\bar{x}^{k}-y\right|^{n+s p}} d y \\
=C_{n, s, p} P . V \cdot \int_{B_{2 r_{k}}\left(x^{k}\right)} \frac{G\left(u\left(\bar{x}^{k}\right)-u(y)\right)+G\left(\varepsilon_{k} \Phi_{r_{k}}\left(\bar{x}^{k}\right)-\varepsilon_{k} \Phi_{r_{k}}(y)\right)}{\left|\bar{x}^{k}-y\right|^{n+s p}} d y \\
\quad+C_{n, s, p} \int_{\mathbb{R}^{n} \backslash B_{2 r_{k}}\left(x^{k}\right)} \frac{G\left(u\left(\bar{x}^{k}\right)-u(y)\right)+G\left(\varepsilon_{k} \Phi_{r_{k}}\left(\bar{x}^{k}\right)\right)}{\left|\bar{x}^{k}-y\right|^{n+s p}} d y \\
=I_{1}+I_{2} .
\end{aligned}
$$

For $I_{1}$, we first notice that

$$
G\left(u\left(\bar{x}^{k}\right)-u(y)\right)+G\left(\varepsilon_{k} \Phi_{r_{k}}\left(\bar{x}^{k}\right)-\varepsilon_{k} \Phi_{r_{k}}(y)\right) \geq 0
$$

due to the strict monotonicity of $G$ and the fact

$$
u\left(\bar{x}^{k}\right)+\varepsilon_{k} \Phi_{r_{k}}\left(\bar{x}^{k}\right)-u(y)-\varepsilon_{k} \Phi_{r_{k}}(y) \geq 0,
$$

for any $y \in B_{2 r_{k}}\left(x^{k}\right)$. Thus

$$
I_{1} \geq 0 .
$$


Now we estimate $I_{2}$, it follows from Lemma 6.1 in Appendix and (2.10) that

$$
\begin{aligned}
I_{2} & =C_{n, s, p} \int_{\mathbb{R}^{n} \backslash B_{2 r_{k}}\left(x^{k}\right)} \frac{G\left(u\left(\bar{x}^{k}\right)-u(y)\right)+G\left(\varepsilon_{k} \Phi_{r_{k}}\left(\bar{x}^{k}\right)\right)}{\left|\bar{x}^{k}-y\right|^{n+s p}} d y \\
& \geq 2^{2-p} C_{n, s, p} \int_{\mathbb{R}^{n} \backslash B_{2 r_{k}}\left(x^{k}\right)} \frac{G\left[u\left(\bar{x}^{k}\right)+\varepsilon_{k} \Phi_{r_{k}}\left(\bar{x}^{k}\right)-u(y)\right]}{\left|\bar{x}^{k}-y\right|^{n+s p}} d y \\
& \geq 2^{2-p} C_{n, s, p} \int_{\left(\mathbb{R}^{n} \backslash B_{2 r_{k}}\left(x^{k}\right)\right) \cap \Sigma} \frac{G\left[u\left(\bar{x}^{k}\right)+\varepsilon_{k} \Phi_{r_{k}}\left(\bar{x}^{k}\right)-u(y)\right]}{\left|\bar{x}^{k}-y\right|^{n+s p}} d y \\
& \geq A^{p-1} 2^{2-p} C_{n, s, p} \int_{\left(\mathbb{R}^{n} \backslash B_{2 r_{k}}\left(x^{k}\right)\right) \cap \Sigma} \frac{1}{\left|\bar{x}^{k}-y\right|^{n+s p}} d y \\
& \geq c_{1} \int_{\Sigma \backslash B_{2 r_{k}}\left(x^{k}\right)} \frac{1}{\left|x^{k}-y\right|^{n+s p}} d y,
\end{aligned}
$$

where the last inequality we have used the fact

$$
\left|\bar{x}^{k}-y\right| \leq\left|\bar{x}^{k}-x^{k}\right|+\left|x^{k}-y\right| \leq \frac{3}{2}\left|x^{k}-y\right| .
$$

We choose $r_{k}=\operatorname{dist}\left(x^{k}, \partial \Sigma\right)$, by (2.1), there exists $j_{0} \geq 1$ such that

$$
\begin{aligned}
I_{2} & \geq c_{1} \int_{\Sigma \backslash B_{2 r_{k}}\left(x^{k}\right)} \frac{1}{\left|x^{k}-y\right|^{n+s p}} d y \\
& \geq c_{1} \sum_{j=j_{0}}^{\infty} \frac{\left|\left(B_{2^{j+1} r_{k}}\left(x^{k}\right) \backslash B_{2^{j} r_{k}}\left(x^{k}\right)\right) \cap \Sigma\right|}{\left(2^{j+1} r_{k}\right)^{n+s p}} \\
& \geq c^{\prime} \sum_{j=j_{0}}^{\infty} \frac{1}{\left(2^{j+1} r_{k}\right)^{s p}}=\frac{2^{-j_{0}} c^{\prime}}{r_{k}^{s p}} .
\end{aligned}
$$

where $c^{\prime}>0$ depending on $c_{0}$ and $c_{1}$.

On the other hand, by (2.2) and (2.9), we deduce that

$$
(-\Delta)_{p}^{s} u\left(\bar{x}^{k}\right) \leq 0
$$

which combining with (2.11), (2.12) and (2.14), yields

$$
\begin{aligned}
& \frac{\varepsilon_{k}^{p-1}}{r_{k}^{s p}}\left[(-\Delta)_{p}^{s} \Phi\right]\left(\frac{\bar{x}^{k}-x^{k}}{r_{k}}\right) \\
& \geq(-\Delta)_{p}^{s} u\left(\bar{x}^{k}\right)+\frac{\varepsilon_{k}^{p-1}}{r_{k}^{s p}}\left[(-\Delta)_{p}^{s} \Phi\right]\left(\frac{\bar{x}^{k}-x^{k}}{r_{k}}\right) \\
& =(-\Delta)_{p}^{s} u\left(\bar{x}^{k}\right)+(-\Delta)_{p}^{s}\left[\varepsilon_{k} \Phi_{r_{k}}\left(\bar{x}^{k}\right)\right] \\
& \geq \frac{2^{-j_{0}} c^{\prime}}{r_{k}^{s p}} .
\end{aligned}
$$

In fact, it is easy to check $\left|\left[(-\Delta)_{p}^{s} \Phi\right]\left(\frac{\bar{x}^{k}-x^{k}}{r_{k}}\right)\right| \leq c$ for $p>2$ (see Lemma 5.2 in Chen and Li [16]). Then we arrive at

$$
\varepsilon_{k}^{p-1} \geq 2^{-j_{0}} c_{1}
$$


Recalling (2.4) and (2.7), we can take $\varepsilon_{k}$ sufficiently small provided $\gamma_{k}$ is close to 1 to derive a contradiction with (2.15), and thus complete the proof of Theorem 2.1.

To prove Theorem 2.3, we also need the following strong maximum principle.

Lemma 2.2. (Strong maximum principle) Let $D$ be an open set in $\mathbb{R}^{n}$, possibly unbounded and disconnected. Assume that both $u$ and $v$ are continuous functions in $C_{l o c}^{1,1}(D) \cap \mathcal{L}_{s p}$ and satisfy

$$
\left\{\begin{array}{l}
(-\Delta)_{p}^{s} u(x)-(-\Delta)_{p}^{s} v(x)=f(u(x))-f(v(x)), x \in D, \\
u(x) \geq v(x), \quad x \in \mathbb{R}^{n} .
\end{array}\right.
$$

where $f$ is a continuous function.

Then either $u(x)>v(x)$, or $u(x) \equiv v(x)$ in $\mathbb{R}^{n}$.

Proof. Let

$$
w(x)=u(x)-v(x)
$$

Assume that there exists $x^{0}$ in $\mathbb{R}^{n}$ such that

$$
w\left(x^{0}\right)=\min _{x \in \mathbb{R}^{n}} w(x)=0 .
$$

It follows from (2.16) that

$$
\begin{aligned}
& (-\Delta)_{p}^{s} u\left(x^{0}\right)-(-\Delta)_{p}^{s} v\left(x^{0}\right) \\
& =C_{n, s, p} P . V . \int_{\mathbb{R}^{n}} \frac{G\left(u\left(x^{0}\right)-u(y)\right)-G\left(v\left(x^{0}\right)-v(y)\right)}{\left|x^{0}-y\right|^{n+s p}} d y .
\end{aligned}
$$

Since

$$
\left[u\left(x^{0}\right)-u(y)\right]-\left[v\left(x^{0}\right)-v(y)\right]=w\left(x^{0}\right)-w(y)=-w(y) \leq 0,
$$

and due to the monotonicity of $G$, we have

$$
G\left(u\left(x^{0}\right)-u(y)\right)-G\left(v\left(x^{0}\right)-v(y)\right) \leq 0 .
$$

Therefore, by (2.16), (2.17) and (2.18), we must have

$$
w(y)=0, \quad \text { for any } y \in \mathbb{R}^{n} .
$$

This completes the proof of the lemma.

We consider

$$
\begin{cases}(-\Delta)_{p}^{s} u(x)=f(u(x)), & x \in \Omega, \\ u(x)>0, & x \in \Omega .\end{cases}
$$

Based on the above two maximum principles, we prove the following theorem.

Theorem 2.3. Let $u \in C_{l o c}^{1,1}(\Omega) \cap \mathcal{L}_{s p}$ be a bounded solution of (2.19) and

$$
u(x)<\mu, \quad x \in \mathbb{R}^{n} \backslash \Omega .
$$

Assume that $f$ is a continuous function satisfying

(a) There exists $\mu>0$ such that $f(t)>0$ on $(0, \mu)$, and $f(t) \leq 0$ for $t \geq \mu$.

Then $u<\mu$ in $\Omega$. 
Proof. Without loss of generality, we always assume that $\mu=1$ in conditions (a)-(c) in the rest of our paper.

Now we first show that $u(x) \leq 1$, for all $x \in \Omega$. Indeed, if $u>1$ somewhere, let $D$ be a component of the set where $u>1$. Notice that $u<1$ in $\mathbb{R}^{n} \backslash \Omega$, let

$$
w_{1}=u-1 \text {. }
$$

Since $f(1)=0, f(u(x)) \leq 0, x \in D$, we have

$$
(-\Delta)_{p}^{s} w_{1}(x) \leq 0, \quad x \in D .
$$

It follows from Theorem 2.1 that

$$
w_{1}(x) \leq 0, \quad x \in D .
$$

Thus $u \leq 1$ in $D$, which contradicts the assumption $u>1$ somewhere. So we derive that

$$
u(x) \leq 1, \quad x \in \Omega .
$$

By strong maximum principle (Lemma 2.2), we conclude that $u<1$ in $\Omega$.

This completes the proof of Theorem 2.3.

\section{The proof of Theorem 3}

In this section, we consider

$$
\begin{cases}(-\Delta)_{p}^{s} u(x)=f(u(x)), & x \in \Omega, \\ u(x)>0, & x \in \Omega .\end{cases}
$$

We prove

Theorem 3.1. Let $u \in C_{\text {loc }}^{1,1}(\Omega) \cap \mathcal{L}_{s p}$ be a bounded solution of (3.1) with

$$
0 \leq u(x)<\mu, \quad x \in \mathbb{R}^{n} \backslash \Omega .
$$

Assume that $f$ is continuous and satisfy condition (a), and for some $0<t_{0}<t_{1}<\mu$,

(b) $f(t) \geq \delta_{0} t$ on $\left[0, t_{0}\right]$ for some $\delta_{0}>0$, and

(c) $f(t)$ is nonincreasing on $\left(t_{1}, \mu\right)$.

Then $u(x) \rightarrow \mu$ uniformly in $\Omega$ as $\operatorname{dist}(x, \partial \Omega) \rightarrow \infty$.

We first prove that the bounded solution of (3.1) is bounded away from zero at points far away from the boundary.

Lemma 3.2. There exist $\varepsilon_{1}, R_{0}>0$ with $R_{0}$ depending only on $n$ and $\delta_{0}$ (recall condition (b)) such that

$$
u(x)>\varepsilon_{1} \quad \text { if } \quad \operatorname{dist}(x, \partial \Omega)>R_{0} .
$$

Proof. Let $\lambda_{1}=\lambda_{1}\left(B_{1}(0)\right)$ be the principle eigenvalue of $(-\Delta)_{p}^{s}$ in $B_{1}(0)$ with Dirichlet boundary condition, assume that $\psi$ be the eigenfunction of $(-\Delta)_{p}^{s}$ in $B_{1}(0)$, i.e.,

with $\max _{x \in B_{1}} \psi(x)=\psi(0)=1$.

$$
\left\{\begin{array}{l}
(-\Delta)_{p}^{s} \psi(x)=\lambda_{1} \psi(x), \psi(x)>0, x \in B_{1}(0), \\
\psi(x)=0, \quad x \in \mathbb{R}^{n} \backslash B_{1}(0),
\end{array}\right.
$$


Define

$$
\psi_{R}(x)=\psi\left(\frac{x}{R}\right), \quad \psi_{\varepsilon, R}(x)=\varepsilon \psi_{R}(x) .
$$

Then, it is obvious that $\psi_{\varepsilon, R}(0)=\varepsilon \psi(0)=\varepsilon$. For $\varepsilon \in\left(0, t_{0}\right]$, there exists $R_{0}$ sufficiently large such that $\frac{\lambda_{1}}{R_{0}^{s p}}<\delta_{0}$. For simplicity, we use $R$ instead of $R_{0}$, then

$$
\begin{aligned}
(-\Delta)_{p}^{s} \psi_{\varepsilon, R}(x) & =\frac{\varepsilon}{R^{s p}}\left[(-\Delta)_{p}^{s} \psi\right]\left(\frac{x}{R}\right) \\
& =\frac{\lambda_{1} \varepsilon}{R^{s p}} \psi_{R}(x) \\
& \leq \delta_{0}\left(\varepsilon \psi_{R}(x)\right) \\
& \leq f\left(\psi_{\varepsilon, R}(x)\right)
\end{aligned}
$$

where the last inequality is due to the condition $f(t) \geq \delta_{0} t$ for some $t \in\left[0, t_{0}\right]$.

It follows from (3.1) that

$$
\begin{aligned}
(-\Delta)_{p}^{s} u(x)-(-\Delta)_{p}^{s} \psi_{\varepsilon, R}(x) & =f(u(x))-\frac{\lambda_{1}}{R^{s p}} \psi_{\varepsilon, R}(x) \\
& \geq f(u(x))-f\left(\psi_{\varepsilon, R}(x)\right) .
\end{aligned}
$$

For $y^{0} \in \Omega$ with $\operatorname{dist}\left(y^{0}, \partial \Omega\right)>R$, we choose $\varepsilon_{0}$ small enough such that

$$
\varepsilon_{0}<\inf _{x \in B_{R}\left(y^{0}\right)} u(x)
$$

Then, set $\varepsilon_{1}=\min \left\{\varepsilon_{0}, t_{0}\right\}$, we have

$$
u(x)>\varepsilon_{1} \psi_{R}\left(x-y^{0}\right)=\psi_{\varepsilon_{1}, R}\left(x-y^{0}\right), \quad x \in \overline{B_{R}\left(y^{0}\right)} .
$$

For $t \in[0,1]$ and $y \in \Omega$ with $\operatorname{dist}(y, \partial \Omega)>R$, let $y_{t}=t y+(1-t) y^{0}$ and

$$
w_{t}(x)=u(x)-\psi_{\varepsilon_{1}, R}\left(x-y_{t}\right), \quad x \in \overline{B_{R}\left(y_{t}\right)} .
$$

It follows from (3.3) that

$$
w_{0}(x)>0, \quad x \in \overline{B_{R}\left(y^{0}\right)},
$$

and

$$
w_{t}(x)>0, \quad x \in \partial B_{R}\left(y_{t}\right) .
$$

Now we will prove that

$$
w_{t}(x)>0 \text {, for any } x \in B_{R}\left(y_{t}\right) \text {. }
$$

Suppose on the contrary that there is a fist $t$ such that the graph of $\psi_{\varepsilon_{1}, R}\left(\cdot-y_{t}\right)$ touches that of $u$ at some point $\bar{x}_{R} \in \overline{B_{R}\left(y_{t}\right)}$. Then, from (3.4), we deduce that $\bar{x}_{R} \in B_{R}\left(y_{t}\right)$ and

$$
w_{t}\left(\bar{x}_{R}\right)=0 .
$$


On the other hand,

$$
\begin{aligned}
(-\Delta)_{p}^{s} u\left(\bar{x}_{R}\right)-(-\Delta)_{p}^{s} \psi_{\varepsilon_{1}, R}\left(\bar{x}_{R}-y_{t}\right) \\
=C_{n, s, p} P . V \cdot \int_{\mathbb{R}^{n}} \frac{G\left(u\left(\bar{x}_{R}\right)-u(z)\right)-G\left(\psi_{\varepsilon_{1}, R}\left(\bar{x}_{R}-y_{t}\right)-\psi_{\varepsilon_{1}, R}\left(z-y_{t}\right)\right)}{\left|\bar{x}_{R}-z\right|^{n+s p}} d z \\
=C_{n, s, p} P . V \cdot \int_{B_{R}\left(y_{t}\right)} \frac{G\left(u\left(\bar{x}_{R}\right)-u(z)\right)-G\left(\psi_{\varepsilon_{1}, R}\left(\bar{x}_{R}-y_{t}\right)-\psi_{\varepsilon_{1}, R}\left(z-y_{t}\right)\right)}{\left|\bar{x}_{R}-z\right|^{n+s p}} d z \\
\quad+C_{n, s, p} \int_{\mathbb{R}^{n} \backslash B_{R}\left(y_{t}\right)} \frac{G\left(u\left(\bar{x}_{R}\right)-u(z)\right)-G\left(\psi_{\varepsilon_{1}, R}\left(\bar{x}_{R}-y_{t}\right)\right)}{\left|\bar{x}_{R}-z\right|^{n+s p}} d z \\
=C_{n, s, p}\left\{I_{1}+I_{2}\right\} .
\end{aligned}
$$

We first estimate $I_{1}$, for $z \in B_{R}\left(x_{R}\right)$, we have

$$
G\left(u\left(\bar{x}_{R}\right)-u(z)\right)-G\left(\psi_{\varepsilon_{1}, R}\left(\bar{x}_{R}-y_{t}\right)-\psi_{\varepsilon_{1}, R}\left(z-y_{t}\right)\right) \leq 0 \text { but } \not \equiv 0,
$$

due to the monotonicity of $G$ and the fact that

$$
\left.\left[u\left(\bar{x}_{R}\right)-u(z)\right]-\left[\psi_{\varepsilon_{1}, R}\left(\bar{x}_{R}-y_{t}\right)-\psi_{\varepsilon_{1}, R}\left(z-y_{t}\right)\right)\right]=w_{t}\left(\bar{x}_{R}\right)-w_{t}(z) \leq 0 \text { but } \not \equiv 0 .
$$

One immediately has

$$
I_{1}<0 \text {. }
$$

For $I_{2}, z \in \mathbb{R}^{n} \backslash B_{R}\left(x_{R}\right)$, we also can deduce

$$
G\left(u\left(\bar{x}_{R}\right)-u(z)\right)-G\left(\psi_{\varepsilon_{1}, R}\left(\bar{x}_{R}-y_{t}\right)\right) \leq 0 \text { but } \not \equiv 0 .
$$

Thus $I_{2}<0$, it follows that

$$
(-\Delta)_{p}^{s} u\left(\bar{x}_{R}\right)-(-\Delta)_{p}^{s} \psi_{\varepsilon_{1}, R}\left(\bar{x}_{R}-y_{t}\right)<0 .
$$

On the other hand, by (3.2), we obtain

$$
\begin{aligned}
(-\Delta)_{p}^{s} u\left(\bar{x}_{R}\right)-(-\Delta)_{p}^{s} \psi_{\varepsilon_{1}, R}\left(\bar{x}_{R}-y_{t}\right) & \geq f\left(u\left(\bar{x}_{R}\right)\right)-f\left(\psi_{\varepsilon_{1}, R}\left(\bar{x}_{R}-y_{t}\right)\right) \\
& =0 .
\end{aligned}
$$

It follows from (3.6), (3.7) and (3.8) that (3.5) must be valid. Let $t=1$, we obtain

$$
u(x)>\varepsilon_{1} \psi_{R}(x-y), \text { for any } x \in \overline{B_{R}(y)} .
$$

In particular, $x=y$, it yields that

$$
u(y)>\varepsilon_{1}, \text { for all } y \in \Omega \text { with } \operatorname{dist}(y, \partial \Omega)>R .
$$

This completes the proof of the lemma.

Now, we prove Theorem 3.1. Let

$$
\phi(x)= \begin{cases}c_{n} e^{\frac{1}{|x|^{2}-1}}, & |x|<1 \\ 0, & |x| \geq 1\end{cases}
$$

We choose $c_{n}$ such that $\phi(0)=1$. Set

$$
\phi_{R}(x)=\phi\left(\frac{x-x_{R}}{R}\right),
$$

where $x_{R}$ satisfies $\operatorname{dist}\left(x_{R}, \partial \Omega\right)>2 R$ and $B_{R}\left(x_{R}\right) \subset \Omega$. 
It is obvious that $\phi_{R}\left(x_{R}\right)=\max _{x \in B_{R}\left(x_{R}\right)} \phi_{R}(x)=\phi(0)=1$, and $\phi_{R}$ satisfies

$$
\begin{cases}(-\Delta)_{p}^{s} \phi_{R}(x) \leq \frac{C}{R^{s p}}, & x \in B_{R}\left(x_{R}\right), \\ \phi_{R}(x)=0, & x \in \mathbb{R}^{n} \backslash B_{R}\left(x_{R}\right) .\end{cases}
$$

As a consequence, it follows from (3.1) that

$$
(-\Delta)_{p}^{s} u(x)-(-\Delta)_{p}^{s} \phi_{R}(x) \geq f(u(x))-\frac{C}{R^{s p}} .
$$

Let $w_{R}(x)=u(x)-\phi_{R}(x)$, since $\phi_{R}\left(x_{R}\right)=1$ and notice that $u(x)<1, x \in B_{R}\left(x_{R}\right)$. We infer that there exists $\bar{x}_{R}$ such that

$$
w_{R}\left(\bar{x}_{R}\right)=\min _{x \in B_{R}\left(x_{R}\right)} w_{R}(x)<0,
$$

which implies

$$
u\left(\bar{x}_{R}\right)-\phi_{R}\left(\bar{x}_{R}\right) \leq u\left(x_{R}\right)-\phi_{R}\left(x_{R}\right) .
$$

It follows immediately that

$$
u\left(\bar{x}_{R}\right) \leq u\left(x_{R}\right)-\left(\phi_{R}\left(x_{R}\right)-\phi_{R}\left(\bar{x}_{R}\right)\right) \leq u\left(x_{R}\right) .
$$

On the other hand,

$$
\begin{aligned}
( & \Delta)_{p}^{s} u\left(\bar{x}_{R}\right)-(-\Delta)_{p}^{s} \phi_{R}\left(\bar{x}_{R}\right) \\
= & C_{n, s, p} P . V \cdot \int_{\mathbb{R}^{n}} \frac{G\left(u\left(\bar{x}_{R}\right)-u(y)\right)-G\left(\phi_{R}\left(\bar{x}_{R}\right)-\phi_{R}(y)\right)}{\left|\bar{x}_{R}-y\right|^{n+s p}} d y \\
= & C_{n, s, p} P . V \cdot \int_{B_{R}\left(x_{R}\right)} \frac{G\left(u\left(\bar{x}_{R}\right)-u(y)\right)-G\left(\phi_{R}\left(\bar{x}_{R}\right)-\phi_{R}(y)\right)}{\left|\bar{x}_{R}-y\right|^{n+s p}} d y \\
& +C_{n, s, p} \int_{\mathbb{R}^{n} \backslash B_{R}\left(x_{R}\right)} \frac{G\left(u\left(\bar{x}_{R}\right)-u(y)\right)-G\left(\phi_{R}\left(\bar{x}_{R}\right)\right)}{\left|\bar{x}_{R}-y\right|^{n+s p}} d y \\
= & C_{n, s, p}\left\{I_{1}+I_{2}\right\} .
\end{aligned}
$$

For $I_{1}, y \in B_{R}\left(x_{R}\right)$, we have

$$
G\left(u\left(\bar{x}_{R}\right)-u(y)\right)-G\left(\phi_{R}\left(\bar{x}_{R}\right)-\phi_{R}(y)\right) \leq 0 \text { but } \not \equiv 0,
$$

due to the monotonicity of $G$ and the fact that

$$
\left.\left[u\left(\bar{x}_{R}\right)-u(y)\right]-\left[\phi_{R}\left(\bar{x}_{R}\right)-\phi_{R}(y)\right)\right]=w\left(\bar{x}_{R}\right)-w(y) \leq 0 \text { but } \not \equiv 0 .
$$

One immediately has

$$
I_{1}<0 .
$$

For $I_{2}, y \in \mathbb{R}^{n} \backslash B_{R}\left(x_{R}\right)$, we also can deduce

$$
G\left(u\left(\bar{x}_{R}\right)-u(y)\right)-G\left(\phi_{R}\left(\bar{x}_{R}\right)\right) \leq 0 \text { but } \not \equiv 0 .
$$

Thus $I_{2}<0$, it follows that

$$
(-\Delta)_{p}^{s} u\left(\bar{x}_{R}\right)-(-\Delta)_{p}^{s} \phi_{R}\left(\bar{x}_{R}\right)<0,
$$

combining this with (3.9) gives that

$$
f\left(u\left(\bar{x}_{R}\right)\right)<\frac{C}{R^{s p}} .
$$


Hence, we obtain that

$$
f\left(u\left(\bar{x}_{R}\right)\right) \rightarrow 0, \text { as } R \rightarrow \infty
$$

Now we claim that

$$
u\left(\bar{x}_{R}\right) \rightarrow 1, \text { as } R \rightarrow \infty .
$$

In fact, by Lemma 3.2 , we have $u\left(\bar{x}_{R}\right) \geq \varepsilon_{1}\left(\varepsilon_{1}>0\right)$ as $\bar{x}_{R}$ away from boundary, assume that $u\left(\bar{x}_{R}\right) \in\left[0, t_{0}\right]$, we get from condition $(\mathrm{b})$,

$$
f\left(u\left(\bar{x}_{R}\right)\right) \geq \delta_{0} u\left(\bar{x}_{R}\right) \geq \delta_{0} \varepsilon_{1}
$$

for some $\delta_{0}>0$. Meanwhile, by condition (a), $f$ is a continuous function in $\mathbb{R}$ and $f(t)>0$ in $(0,1)$, we have

$$
\inf _{t \in\left[t_{0}, t_{1}\right]} f(t)=c_{0}>0 .
$$

Therefore, we derive from (3.11), (3.13) and (3.14) that $u\left(\bar{x}_{R}\right)$ must fall in open interval $\left(t_{1}, 1\right)$, in which $f(t)$ is nonincreasing due to condition (c). Hence (3.12) must be valid. It follows from (3.10) that

$$
1>u\left(x_{R}\right) \geq u\left(\bar{x}_{R}\right) \rightarrow 1, \text { as } R \rightarrow \infty .
$$

Thus, we obtain that $u\left(x_{R}\right) \rightarrow 1$ as $R \rightarrow \infty$.

This completes the proof of Theorem 3.1.

\section{The Proof of Theorem 4}

In [39], Li considered the following equation,

$$
\begin{cases}(-\Delta)_{p}^{s} u(x)=g(x, u), u>0, & x \in \Gamma, \\ u(x) \leq 0, & x \in \mathbb{R}^{n} \backslash \Gamma .\end{cases}
$$

Based on Jin and Li [35] (the boundary Hölder regularity) and Brasco, Lindgren and Schikorra [7] (the interior Hölder regularity), Li [39] obtained the following uniform Hölder norm estimate in $\mathbb{R}^{n}$ for the fractional p-Laplacian.

Lemma 4.1. (The uniform Hölder norm estimate) Assume that $\Gamma$ is a domain (possibly unbounded) with the uniform two-sided ball condition, and $u \in C_{l o c}^{1,1} \cap \mathcal{L}_{s p}$ is a bounded solution of (4.1). If $g(x, u)$ is bounded, then there exists $\alpha \in(0, s)$ such that $u \in C^{\alpha}\left(\mathbb{R}^{n}\right)$. Moreover,

$$
[u]_{C^{\alpha}\left(\mathbb{R}^{n}\right)} \leq C\left(1+\|u\|_{L^{\infty}(\Gamma)}+\|g\|_{L^{\infty}(\Gamma)}^{\frac{1}{p-1}}\right)
$$

where $C$ is a constant depending on $\alpha, s, p, \Gamma$.

Based on this uniform estimate, we apply the sliding method to derive the monotonicity and uniqueness of solutions for

$$
\begin{cases}(-\Delta)_{p}^{s} u(x)=f(u(x)), & x \in \Omega, \\ u(x)>0, & x \in \Omega, \\ u(x)=0, & x \in \mathbb{R}^{n} \backslash \Omega,\end{cases}
$$

where $\Omega$ satisfies the uniform two-sided ball condition. 
Theorem 4.2. Let $u \in C_{\text {loc }}^{1,1}(\Omega) \cap \mathcal{L}_{s p}$ be a bounded solution of (4.2). Assume that $f$ is a continuous function and satisfies conditions (a)-(c) for some $0<t_{0}<t_{1}<\mu$.

Then $u$ is strictly monotone increasing in $x_{n}$.

Furthermore the bounded solution of (4.2) is unique.

Proof. We will carry out the proof of Theorem 4.2 in three steps.

For $\tau \geq 0$, denote

$$
u_{\tau}(x):=u\left(x+\tau e_{n}\right), \quad w_{\tau}(x):=u(x)-u_{\tau}(x),
$$

where $e_{n}=(0,0, \cdots, 1)$.

In step 1, we will show that for $\tau$ sufficiently large, we have

$$
w_{\tau}(x) \leq 0, \quad x \in \mathbb{R}^{n} .
$$

This provides the starting point for the sliding method. Then in step 2, we decrease $\tau$ continuously as long as (4.3) holds to its limiting position. Define

$$
\tau_{0}:=\inf \left\{\tau>0 \mid w_{\tau}(x) \leq 0, \forall x \in \mathbb{R}^{n}\right\} .
$$

We will show that $\tau_{0}=0$. Then we deduce that the solution $u$ must be strictly monotone increasing in $x_{n}$. In step 3, we will prove the uniqueness by constructing the sub-solution.

We now show the details in the three steps.

Step 1. We show that for $\tau$ sufficiently large, we have

$$
w_{\tau}(x) \leq 0, \quad x \in \mathbb{R}^{n} .
$$

For $h>0$, define

$$
\Omega_{h}:=\left\{x \in \mathbb{R}^{n} \mid \varphi\left(x^{\prime}\right)<x_{n}<\varphi\left(x^{\prime}\right)+h\right\} .
$$

By Theorem 3.1, there exists an $M_{0}>0$ large enough such that for $\tau \geq M_{0}$,

$$
u_{\tau}(x) \in\left(t_{1}, 1\right), \forall x \in \Omega_{h} .
$$

Suppose (4.4) is violated, there exists a constant $A>0$ such that

$$
\sup _{x \in \mathbb{R}^{n}} w_{\tau}(x)=A,
$$

hence there exists a sequence $\left\{x^{k}\right\}$ in $\mathbb{R}^{n}$ such that

$$
w_{\tau}\left(x^{k}\right) \rightarrow A, \text { as } k \rightarrow \infty .
$$

Since $u=0$ in $\mathbb{R}^{n} \backslash \bar{\Omega}$, it yields that

$$
w_{\tau}(x) \leq 0, \quad \forall x \in \mathbb{R}^{n} \backslash \bar{\Omega} .
$$

Moreover, thanks to Theorem 3.1, there exists an $M>M_{0}>0$ such that the sequence $x^{k} \in \Omega_{M}$. Similar to the argument as Theorem 2.1, there exists $\bar{x}^{k} \in B_{1}\left(x^{k}\right)$ such that

$$
w_{\tau}\left(\bar{x}^{k}\right)+\varepsilon_{k} \Phi_{1}\left(\bar{x}^{k}\right)=\max _{x \in B_{1}\left(x^{k}\right)}\left[w_{\tau}(x)+\varepsilon_{k} \Phi_{1}(x)\right]=\max _{x \in \mathbb{R}^{n}}\left[w_{\tau}(x)+\varepsilon_{k} \Phi_{1}(x)\right]>A,
$$

where the definition of $\Phi_{r_{k}}$ is the same as (2.6) with $r_{k}=1$. 
It follows from Lemma 6.2 in Appendix and the monotonicity of $f(t)$ for $t \in\left(t_{1}, 1\right)$ that

$$
\begin{aligned}
& (-\Delta)_{p}^{s}\left(u+\varepsilon_{k} \Phi_{1}\right)\left(\bar{x}^{k}\right)-(-\Delta)_{p}^{s} u_{\tau}\left(\bar{x}^{k}\right) \\
& =(-\Delta)_{p}^{s}\left(u+\varepsilon_{k} \Phi_{1}\right)\left(\bar{x}^{k}\right)-(-\Delta)_{p}^{s} u\left(\bar{x}^{k}\right)+(-\Delta)_{p}^{s} u\left(\bar{x}^{k}\right)-(-\Delta)_{p}^{s} u_{\tau}\left(\bar{x}^{k}\right) \\
& \leq f\left(u\left(\bar{x}^{k}\right)\right)-f\left(u_{\tau}\left(\bar{x}^{k}\right)\right)+\varepsilon_{k} C_{\delta}+C \delta^{p(1-s)} \\
& \leq \varepsilon_{k} C_{\delta}+C \delta^{p(1-s)}
\end{aligned}
$$

On the other hand, we calculate

$$
\begin{aligned}
( & \Delta)_{p}^{s}\left(u+\varepsilon_{k} \Phi_{1}\right)\left(\bar{x}^{k}\right)-(-\Delta)_{p}^{s} u_{\tau}\left(\bar{x}^{k}\right) \\
= & C_{n, s, p} P . V \cdot \int_{\mathbb{R}^{n}} \frac{G\left(u\left(\bar{x}^{k}\right)+\varepsilon_{k} \Phi_{1}\left(\bar{x}^{k}\right)-u(y)-\varepsilon_{k} \Phi_{1}(y)\right)-G\left(u_{\tau}\left(\bar{x}^{k}\right)-u_{\tau}(y)\right)}{\left|\bar{x}^{k}-y\right|^{n+s p}} d y \\
= & C_{n, s, p} P . V \cdot \int_{B_{2}\left(x^{k}\right)} \frac{G\left(u\left(\bar{x}^{k}\right)+\varepsilon_{k} \Phi_{1}\left(\bar{x}^{k}\right)-u(y)-\varepsilon_{k} \Phi_{1}(y)\right)-G\left(u_{\tau}\left(\bar{x}^{k}\right)-u_{\tau}(y)\right)}{\left|\bar{x}^{k}-y\right|^{n+s p}} d y \\
& +C_{n, s, p} \int_{\mathbb{R}^{n} \backslash B_{2}\left(x^{k}\right)} \frac{G\left(u\left(\bar{x}^{k}\right)+\varepsilon_{k} \Phi_{1}\left(\bar{x}^{k}\right)-u(y)-\varepsilon_{k} \Phi_{1}(y)\right)-G\left(u_{\tau}\left(\bar{x}^{k}\right)-u_{\tau}(y)\right)}{\left|\bar{x}^{k}-y\right|^{n+s p}} d y \\
= & I_{1}+I_{2} .
\end{aligned}
$$

For $I_{1}$, we first notice that

$$
G\left(u\left(\bar{x}^{k}\right)+\varepsilon_{k} \Phi_{1}\left(\bar{x}^{k}\right)-u(y)-\varepsilon_{k} \Phi_{1}(y)\right)-G\left(u_{\tau}\left(\bar{x}^{k}\right)-u_{\tau}(y)\right) \geq 0
$$

due to the strict monotonicity of $G$ and the fact

$$
\begin{gathered}
u\left(\bar{x}^{k}\right)+\varepsilon_{k} \Phi_{1}\left(\bar{x}^{k}\right)-u(y)-\varepsilon_{k} \Phi_{1}(y)-\left(u_{\tau}\left(\bar{x}^{k}\right)-u_{\tau}(y)\right) \\
=w_{\tau}\left(\bar{x}^{k}\right)+\varepsilon_{k} \Phi_{1}\left(\bar{x}^{k}\right)-\left(w_{\tau}(y)+\varepsilon_{k} \Phi_{1}(y)\right) \geq 0,
\end{gathered}
$$

for any $y \in B_{2}\left(x^{k}\right)$. Thus

$$
I_{1} \geq 0 \text {. }
$$

Now we estimate $I_{2}$, one can infer from Lemma 6.1 in Appendix and (4.5) that

$$
\begin{aligned}
I_{2} & =C_{n, s, p} \int_{\mathbb{R}^{n} \backslash B_{2}\left(x^{k}\right)} \frac{G\left(u\left(\bar{x}^{k}\right)+\varepsilon_{k} \Phi_{1}\left(\bar{x}^{k}\right)-u(y)-\varepsilon_{k} \Phi_{1}(y)\right)-G\left(u_{\tau}\left(\bar{x}^{k}\right)-u_{\tau}(y)\right)}{\left|\bar{x}^{k}-y\right|^{n+s p}} d y \\
& \geq 2^{2-p} C_{n, s, p} \int_{\mathbb{R}^{n} \backslash B_{2}\left(x^{k}\right)} \frac{G\left[w_{\tau}\left(\bar{x}^{k}\right)+\varepsilon_{k} \Phi_{1}\left(\bar{x}^{k}\right)-u(y)\right]}{\left|\bar{x}^{k}-y\right|^{n+s p}} d y \\
& \geq 2^{2-p} C_{n, s, p} \int_{\left(\mathbb{R}^{n} \backslash B_{2}\left(x^{k}\right)\right) \cap\left(\mathbb{R}^{n} \backslash \bar{\Omega}\right)} \frac{G\left[w_{\tau}\left(\bar{x}^{k}\right)+\varepsilon_{k} \Phi_{1}\left(\bar{x}^{k}\right)-w_{\tau}(y)\right]}{\left|\bar{x}^{k}-y\right|^{n+s p}} d y \\
& \geq A^{p-1} 2^{2-p} C_{n, s, p} \int_{\left(\mathbb{R}^{n} \backslash B_{2}\left(x^{k}\right)\right) \cap\left(\mathbb{R}^{n} \backslash \bar{\Omega}\right)} \frac{1}{\left|\bar{x}^{k}-y\right|^{n+s p}} d y \\
& \geq c_{1} \int_{\left(\mathbb{R}^{n} \backslash B_{2}\left(x^{k}\right)\right) \cap\left(\mathbb{R}^{n} \backslash \bar{\Omega}\right)} \frac{1}{\left|x^{k}-y\right|^{n+s p}} d y,
\end{aligned}
$$

where in the last inequality we have used the fact

$$
\left|\bar{x}^{k}-y\right| \leq\left|\bar{x}^{k}-x^{k}\right|+\left|x^{k}-y\right| \leq \frac{3}{2}\left|x^{k}-y\right| .
$$


As a consequence, we get

$$
I_{2} \geq c_{1} \int_{\left(\mathbb{R}^{n} \backslash B_{2}\left(x^{k}\right)\right) \cap\left(\mathbb{R}^{n} \backslash \bar{\Omega}\right)} \frac{1}{\left|x^{k}-y\right|^{n+s p}} d y \geq c_{M}>0 .
$$

where $x^{k} \in \Omega_{M}$.

It follows from (4.6), (4.7), (4.8), (4.9) that

$$
\varepsilon_{k} C_{\delta}+C \delta^{p(1-s)} \geq c_{M} .
$$

Choosing $\delta=\left(\frac{c_{M}}{2 C}\right)^{\frac{1}{p(1-s)}}$, we arrive at

$$
\varepsilon_{k} C_{\delta} \geq \frac{c_{M}}{2}
$$

Since the left hand side of (4.10) must go to zero as $\varepsilon_{k} \rightarrow 0(k \rightarrow \infty)$, which contradicts the right hand side of (4.10). Therefore, (4.4) must be true for sufficiently large $\tau$.

Step 2. Now we prove that for $\forall \tau>0$,

$$
w_{\tau}(x)<0, \quad \forall x \in \mathbb{R}^{n} .
$$

Step 1 provides a starting point from which we can decrease $\tau$ continuously from $\tau \geq N$ as long as (4.4) holds, define

$$
\tau_{0}:=\inf \left\{\tau>0 \mid w_{\tau}(x) \leq 0, \forall x \in \mathbb{R}^{n}\right\} .
$$

We show that

$$
\tau_{0}=0 .
$$

Suppose on the contrary $\tau_{0}>0$. By continuity, we see that $w_{\tau_{0}}(x) \leq 0$. One can infer from strong maximum principle (Lemma 2.2) that

$$
w_{\tau_{0}}(x)<0, \forall x \in \mathbb{R}^{n} .
$$

Then the following two cases may occur.

Case 1. Suppose that

$$
\sup _{x \in \Omega_{M}} w_{\tau_{0}}(x)=0
$$

where $M$ is the same as Step 1 .

Then there exists a sequence $\left\{x^{k}\right\}$ in $\Omega_{M}$ such that

$$
w_{\tau_{0}}\left(x^{k}\right) \rightarrow 0, \text { as } k \rightarrow \infty .
$$

Similar to the argument as Theorem 2.1, by (4.13), then there exists $\bar{x}^{k} \in B_{1}\left(x^{k}\right)$ such that

$$
w_{\tau_{0}}\left(\bar{x}^{k}\right)+\varepsilon_{k} \Phi_{1}\left(\bar{x}^{k}\right)=\max _{x \in B_{1}\left(x^{k}\right)}\left[w_{\tau_{0}}(x)+\varepsilon_{k} \Phi_{1}(x)\right]=\max _{x \in \mathbb{R}^{n}}\left[w_{\tau_{0}}(x)+\varepsilon_{k} \Phi_{1}(x)\right]>0 .
$$

where the definition of $\Phi_{1}$ is the same as (2.6) with $r_{k}=1$.

By (4.14), we can deduce that

$$
0>w_{\tau_{0}}\left(\bar{x}^{k}\right) \geq w_{\tau_{0}}\left(x^{k}\right)+\varepsilon_{k} \Phi_{1}\left(x^{k}\right)-\varepsilon_{k} \Phi_{1}\left(\bar{x}^{k}\right) \geq w_{\tau_{0}}\left(x^{k}\right) \rightarrow 0, \text { as } k \rightarrow \infty,
$$

which implies that

$$
w_{\tau_{0}}\left(\bar{x}^{k}\right) \rightarrow 0, \text { as } k \rightarrow \infty .
$$

It follows from Lemma 6.2 in Appendix that 


$$
\begin{aligned}
& (-\Delta)_{p}^{s}\left(u+\varepsilon_{k} \Phi_{1}\right)\left(\bar{x}^{k}\right)-(-\Delta)_{p}^{s} u_{\tau_{0}}\left(\bar{x}^{k}\right) \\
& =(-\Delta)_{p}^{s}\left(u+\varepsilon_{k} \Phi_{1}\right)\left(\bar{x}^{k}\right)-(-\Delta)_{p}^{s} u\left(\bar{x}^{k}\right)+(-\Delta)_{p}^{s} u\left(\bar{x}^{k}\right)-(-\Delta)_{p}^{s} u_{\tau_{0}}\left(\bar{x}^{k}\right) \\
& \leq f\left(u\left(\bar{x}^{k}\right)\right)-f\left(u_{\tau_{0}}\left(\bar{x}^{k}\right)\right)+\varepsilon_{k} C_{\delta}+C \delta^{p(1-s)} .
\end{aligned}
$$

On the other hand, we calculate

$$
\begin{aligned}
(-\Delta)_{p}^{s}\left(u+\varepsilon_{k} \Phi_{1}\right)\left(\bar{x}^{k}\right)-(-\Delta)_{p}^{s} u_{\tau_{0}}\left(\bar{x}^{k}\right) \\
=C_{n, s, p} P . V \cdot \int_{\mathbb{R}^{n}} \frac{G\left(u\left(\bar{x}^{k}\right)+\varepsilon_{k} \Phi_{1}\left(\bar{x}^{k}\right)-u(y)-\varepsilon_{k} \Phi_{1}(y)\right)-G\left(u_{\tau_{0}}\left(\bar{x}^{k}\right)-u_{\tau_{0}}(y)\right)}{\left|\bar{x}^{k}-y\right|^{n+s p}} d y \\
=C_{n, s, p} P . V . \int_{B_{2}\left(x^{k}\right)} \frac{G\left(u\left(\bar{x}^{k}\right)+\varepsilon_{k} \Phi_{1}\left(\bar{x}^{k}\right)-u(y)-\varepsilon_{k} \Phi_{1}(y)\right)-G\left(u_{\tau_{0}}\left(\bar{x}^{k}\right)-u_{\tau_{0}}(y)\right)}{\left|\bar{x}^{k}-y\right|^{n+s p}} d y \\
\quad+C_{n, s, p} \int_{\mathbb{R}^{n} \backslash B_{2}\left(x^{k}\right)} \frac{G\left(u\left(\bar{x}^{k}\right)+\varepsilon_{k} \Phi_{1}\left(\bar{x}^{k}\right)-u(y)-\varepsilon_{k} \Phi_{1}(y)\right)-G\left(u_{\tau_{0}}\left(\bar{x}^{k}\right)-u_{\tau_{0}}(y)\right)}{\left|\bar{x}^{k}-y\right|^{n+s p}} d y \\
=I_{1}+I_{2} .
\end{aligned}
$$

For $I_{1}$, we first notice that

$$
G\left(u\left(\bar{x}^{k}\right)+\varepsilon_{k} \Phi_{1}\left(\bar{x}^{k}\right)-u(y)-\varepsilon_{k} \Phi_{1}(y)\right)-G\left(u_{\tau_{0}}\left(\bar{x}^{k}\right)-u_{\tau_{0}}(y)\right) \geq 0
$$

due to the strict monotonicity of $G$ and the fact

$$
\begin{aligned}
& u\left(\bar{x}^{k}\right)+\varepsilon_{k} \Phi_{1}\left(\bar{x}^{k}\right)-u(y)-\varepsilon_{k} \Phi_{1}(y)-\left(u_{\tau_{0}}\left(\bar{x}^{k}\right)-u_{\tau_{0}}(y)\right) \\
& =w_{\tau_{0}}\left(\bar{x}^{k}\right)+\varepsilon_{k} \Phi_{1}\left(\bar{x}^{k}\right)-\left(w_{\tau_{0}}(y)+\varepsilon_{k} \Phi_{1}(y)\right) \geq 0,
\end{aligned}
$$

for any $y \in B_{2}\left(x^{k}\right)$. Thus

$$
I_{1} \geq 0 .
$$

Now we estimate $I_{2}$, one can infer from Lemma 6.1] in Appendix and (4.14) that

$$
\begin{aligned}
I_{2} & =C_{n, s, p} \int_{\mathbb{R}^{n} \backslash B_{2}\left(x^{k}\right)} \frac{G\left(u\left(\bar{x}^{k}\right)+\varepsilon_{k} \Phi_{1}\left(\bar{x}^{k}\right)-u(y)-\varepsilon_{k} \Phi_{1}(y)\right)-G\left(u_{\tau_{0}}\left(\bar{x}^{k}\right)-u_{\tau_{0}}(y)\right)}{\left|\bar{x}^{k}-y\right|^{n+s p}} d y \\
& \geq 2^{2-p} C_{n, s, p} \int_{\mathbb{R}^{n} \backslash B_{2}\left(x^{k}\right)} \frac{G\left[w_{\tau_{0}}\left(\bar{x}^{k}\right)+\varepsilon_{k} \Phi_{1}\left(\bar{x}^{k}\right)-w_{\tau_{0}}(y)\right]}{\left|\bar{x}^{k}-y\right|^{n+s p}} d y \\
& \geq c^{\prime} \int_{\mathbb{R}^{n} \backslash B_{2}\left(x^{k}\right)} \frac{G\left[-w_{\tau_{0}}(y)\right]}{\left|x^{k}-y\right|^{n+s p}} d y,
\end{aligned}
$$

where for the last inequality we have used the fact

$$
\left|\bar{x}^{k}-y\right| \leq\left|\bar{x}^{k}-x^{k}\right|+\left|x^{k}-y\right| \leq \frac{3}{2}\left|x^{k}-y\right| .
$$


It follows from (4.17), (4.18) and (4.19) that

$$
\begin{aligned}
& (-\Delta)_{p}^{s}\left(u+\varepsilon_{k} \Phi_{1}\right)\left(\bar{x}^{k}\right)-(-\Delta)_{p}^{s} u_{\tau_{0}}\left(\bar{x}^{k}\right) \\
& \geq c^{\prime} \int_{\mathbb{R}^{n} \backslash B_{2}\left(x^{k}\right)} \frac{G\left[-w_{\tau_{0}}(y)\right]}{\left|x^{k}-y\right|^{n+s p}} d y \\
& =c^{\prime} \int_{\mathbb{R}^{n} \backslash B_{2}(0)} \frac{G\left[-w_{\tau_{0}}\left(y+x^{k}\right)\right]}{|y|^{n+s p}} d y
\end{aligned}
$$

where $x^{k} \in \Omega_{M}$.

As a consequence, by (4.16), we have

$$
f\left(u\left(\bar{x}^{k}\right)\right)-f\left(u_{\tau_{0}}\left(\bar{x}^{k}\right)\right)+\varepsilon_{k} C_{\delta}+C \delta^{p(1-s)} \geq c^{\prime} \int_{\mathbb{R}^{n} \backslash B_{2}(0)} \frac{G\left[-w_{\tau_{0}}\left(y+x^{k}\right)\right]}{|y|^{n+s p}} d y .
$$

Let

$$
w_{\tau_{0}}^{k}(y)=u\left(y+x^{k}\right)-u_{\tau_{0}}\left(y+x^{k}\right) .
$$

From Lemma 4.1, we know that $u$ is uniformly Hölder continuous in $\mathbb{R}^{n}$, hence, $w_{\tau_{0}}^{k}$ is equi-continuous in $\mathbb{R}^{n}$. By the Arzelà-Ascoli theorem, there exists $w_{\tau_{0}}^{\infty}$, such that

$$
w_{\tau_{0}}^{k} \rightarrow w_{\tau_{0}}^{\infty}, \quad \text { as } k \rightarrow \infty \text { uniformly in } \mathbb{R}^{n} .
$$

It follows that the right hand side of inequality (4.21) converges to

$$
c^{\prime} \int_{\mathbb{R}^{n} \backslash B_{2}(0)} \frac{G\left(-w_{\tau_{0}}^{\infty}(y)\right)}{|y|^{n+s p}} d y .
$$

Combining (4.15) with the continuity of $f$ and the fact $\varepsilon_{k} \rightarrow 0(k \rightarrow \infty)$, we see that the left hand side of inequality (4.21) converges to $C \delta^{p(1-s)}(0<\delta<1)$ as $k \rightarrow \infty$. Because of the arbitrariness of $\delta$, we derive that

$$
\int_{\mathbb{R}^{n} \backslash B_{2}(0)} \frac{G\left(-w_{\tau_{0}}^{\infty}(y)\right)}{|y|^{n+s p}} d y \equiv 0,
$$

which implies that

$$
u_{\tau_{0}}^{\infty}(x) \equiv u^{\infty}(x), \quad \forall x \in \mathbb{R}^{n} \backslash B_{2}(0) .
$$

Recall that $u>0$ in $\Omega$ while $u(x) \equiv 0, x \in \mathbb{R}^{n} \backslash \Omega$. Since $x^{k} \in \Omega_{M}$, there exists $x^{o}$ such that $u^{\infty}\left(x^{o}\right)=0$, then by (4.22), we have

$$
0=u_{\tau_{0}}^{\infty}\left(x^{o}\right)=u^{\infty}\left(x^{o}+\tau_{0} e_{n}\right)=u_{\tau_{0}}^{\infty}\left(x^{o}+\tau_{0} e_{n}\right)=u^{\infty}\left(x^{o}+2 \tau_{0} e_{n}\right)=\cdots=u^{\infty}\left(x^{o}+m \tau_{0} e_{n}\right) .
$$

By Theorem 3.1, we deduce that

$$
u^{\infty}\left(x^{o}+m \tau_{0} e_{n}\right) \rightarrow 1 \text { as } m \rightarrow \infty .
$$

This is a contradiction to (4.23)!

Case 2. Suppose that

$$
\sup _{x \in \Omega_{M}} w_{\tau_{0}}(x)<0 .
$$


From Lemma 4.1, we know that $u$ is uniformly Hölder continuous in $\mathbb{R}^{n}$, then for any $\eta \in$ $\left(0, \tau_{0}\right)$ small enough, we get

$$
\sup _{x \in \Omega_{M}} w_{\tau}(x)<0, \quad \forall \tau_{0}-\eta<\tau \leq \tau_{0} .
$$

If there exists a constant $A_{1}>0$ such that

$$
\sup _{x \in \mathbb{R}^{n}} w_{\tau}(x)=A_{1}, \quad \forall \tau_{0}-\eta<\tau \leq \tau_{0},
$$

then there exists a sequence $\left\{x^{k}\right\}$ in $\mathbb{R}^{n}$ such that

$$
w_{\tau}\left(x^{k}\right) \rightarrow A_{1} \text {, as } k \rightarrow \infty .
$$

Since $u=0$ in $\mathbb{R}^{n} \backslash \Omega$, it yields that

$$
w_{\tau}(x) \leq 0, \quad \forall x \in \mathbb{R}^{n} \backslash \Omega .
$$

Thanks to Theorem 3.1 and (4.24), there exists an $M_{1}>M>0$ such that the sequence $\left\{x^{k}\right\}$ is contained in $\Omega_{M_{1}} \backslash \overline{\Omega_{M}}$. For any $x \in \Omega_{M_{1}} \backslash \overline{\Omega_{M}}$, we have

$$
u(x), u_{\tau}(x) \in\left(t_{1}, 1\right),
$$

in which $f(\cdot)$ is non-increasing due to condition (c).

Then, similar to the argument as in Step 1. We can derive

$$
\varepsilon_{k} C_{\delta} \geq \frac{c_{A_{1}}}{2}>0
$$

This is a contradiction as $\varepsilon_{k}(k \rightarrow \infty)$ goes to zero. So, we have

$$
w_{\tau}(x) \leq 0, \quad \forall \tau_{0}-\eta<\tau \leq \tau_{0}, \forall x \in \mathbb{R}^{n} .
$$

This contradicts the definition of $\tau_{0}$.

It follows that

$$
w_{\tau}(x) \leq 0, \quad \forall \tau \geq 0, \forall x \in \mathbb{R}^{n} .
$$

Moreover, by strong maximum principle (Lemma 2.2), we arrive at (4.11).

This implies $u$ is strictly monotone increasing in $x_{n}$.

Step 3. Now we prove the uniqueness. Assume that $u$ and $v$ are two bounded solutions of (4.1). For $\tau \geq 0$, denote

$$
u_{\tau}(x):=u\left(x+\tau e_{n}\right), \quad \tilde{w}_{\tau}(x):=v(x)-u_{\tau}(x),
$$

where $e_{n}=(0,0, \cdots, 1)$.

We first show that for $\tau$ sufficiently large,

$$
\tilde{w}_{\tau}(x) \leq 0, \quad \forall x \in \mathbb{R}^{n} .
$$

The proof of (4.26) is completely similar to Step 1 of the proof of monotonicity, so we omit the details. (4.26) provides a starting point from which we can decrease $\tau$ continuously as long as (4.26) holds.

We prove that

$$
\tilde{w}_{\tau}(x) \leq 0, \forall \tau \geq 0, \forall x \in \mathbb{R}^{n}
$$

Define

$$
\tau_{0}:=\inf \left\{\tau>0 \mid \tilde{w}_{\tau}(x) \leq 0, \forall x \in \mathbb{R}^{n}\right\}
$$


We show that

$$
\tau_{0}=0
$$

Suppose on the contrary $\tau_{0}>0$. Similar to the argument of monotonicity in Step 2, one can deduce that

$$
v^{\infty}(x) \equiv u_{\tau_{0}}^{\infty}(x), \quad \forall x \in \mathbb{R}^{n} \backslash B_{2}(0) .
$$

To finish the proof of the uniqueness, we need the following lemma.

Lemma 4.3. (Li and Zhang [40]) Let $\psi(x)=\left(1-|x|^{2}\right)_{+}^{s}$, then there exists constant $C$, such that

$$
\left|(-\Delta)_{p}^{s} \psi(x)\right| \leq C, \quad \forall x \in B_{1}(0) .
$$

Suppose that $z$ be a point on $\partial \Omega$. Without loss of generality, we may assume that there is a ball $B \subset \Omega$ of radius 1 tangent to $\partial \Omega$ at point $z$. For simplicity of notation, we assume that the center of the ball is the origin.

Let

$$
D=\left\{x \in \Omega \mid \operatorname{dist}(x, \partial \Omega) \geq 2 R_{0}\right\},
$$

where $R_{0}$ is the same as in Lemma 3.2 .

We construct the sub-solution

$$
\underline{u}(x)=u_{D}(x)+\varepsilon \psi(x), \quad x \in B .
$$

where $u_{D}:=u \cdot \chi_{D}$ and $\chi_{D}$ is defined as

$$
\chi_{D}(x)=\left\{\begin{array}{l}
1, x \in D, \\
0, x \in \mathbb{R}^{n} \backslash D .
\end{array}\right.
$$

It follows from (4.30), Lemma 3.2 and Lemma 6.1 in Appendix that for $x \in B$,

$$
\begin{aligned}
(-\Delta)_{p}^{s} \underline{u}(x)= & (-\Delta)_{p}^{s}\left(u_{D}+\varepsilon \psi\right)(x) \\
= & C_{n, s, p} P . V \cdot \int_{\mathbb{R}^{n}} \frac{G\left(u_{D}(x)+\varepsilon \psi(x)-u_{D}(y)-\varepsilon \psi(y)\right)}{|x-y|^{n+s p}} d y \\
= & C_{n, s, p} P . V \cdot\left\{\int_{\mathbb{R}^{n} \backslash B} \frac{G\left(\varepsilon \psi(x)-u_{D}(y)\right)-G(\varepsilon \psi(x))}{|x-y|^{n+s p}} d y\right. \\
& \left.+\int_{\mathbb{R}^{n} \backslash B} \frac{G(\varepsilon \psi(x))}{|x-y|^{n+s p}} d y+\int_{B} \frac{G(\varepsilon \psi(x)-\varepsilon \psi(y))}{|x-y|^{n+s p}} d y\right\} \\
= & \varepsilon^{p-1}(-\Delta)_{p}^{s} \psi(x)+C_{n, s, p} \int_{D} \frac{G(\varepsilon \psi(x)-u(y))-G(\varepsilon \psi(x))}{|x-y|^{n+s p}} d y \\
\leq & \varepsilon^{p-1} C+2^{2-p} C_{n, s, p} \int_{D} \frac{G(-u(y))}{|x-y|^{n+s p}} d y \\
\leq & \varepsilon^{p-1} C-2^{2-p} \varepsilon_{1}^{p-1} C_{n, s, p} \int_{D} \frac{1}{|x-y|^{n+s p}} d y \\
\leq & \varepsilon^{p-1} C-2^{2-p} \varepsilon_{1}^{p-1} C_{n, s, p} C_{R_{0}} .
\end{aligned}
$$


One can choose $\varepsilon \leq \varepsilon_{1}\left[2^{2-p} C_{n, s, p} C_{R_{0}} C^{-1}\right]^{\frac{1}{p-1}}:=\varepsilon_{0}$ such that $(-\Delta)_{p}^{s} \underline{u}(x) \leq 0, x \in B$. Then we fixed $\varepsilon=\frac{\varepsilon_{0}}{2}$, by Lemma 6.3 in Appendix, we arrive at

$$
u(x) \geq \underline{u}(x) \geq \frac{\varepsilon_{0}}{2} \psi(x)=\frac{\varepsilon_{0}}{2}(1+|x|)^{s}(1-|x|)^{s} \geq \frac{\varepsilon_{0}}{2} \delta^{s}(x), \quad \forall x \in B .
$$

For each fixed small $\delta_{1} \in\left(0, \min \left\{\tau_{0}, 1\right\}\right)$, choosing $\left|x_{n}-z_{n}\right|=\delta_{1}$, we derive that

$$
\begin{aligned}
u(x) & \geq \frac{\varepsilon_{0}}{2} \delta^{s}(x) \\
& \geq \frac{\varepsilon_{0}}{2}\left|x_{n}-z_{n}\right|^{s} \\
& =\frac{\varepsilon_{0}}{2} \delta_{1}^{s}>0 .
\end{aligned}
$$

Since $u$ is strictly monotone increasing in $x_{n}$, by (4.31), we infer that

$$
u_{\tau_{0}}(z)>\frac{\varepsilon_{0}}{2} \delta_{1}^{s}>0, \quad \forall z \in \partial \Omega .
$$

Obviously, this property is preserved under translation. Let

$$
\Omega^{k}=\left\{x \mid x+x^{k} \in \Omega\right\} \text { and } \Omega^{\infty}=\lim _{k \rightarrow \infty} \Omega^{k} .
$$

Taking a point $x^{0} \in \partial \Omega^{\infty}$, we deduce from (4.32) that

$$
u_{\tau_{0}}^{\infty}\left(x^{0}\right)>0 \text { but } v^{\infty}\left(x^{0}\right)=0 .
$$

This contradicts (4.29). So we must have $\tau_{0}=0$. This proves (4.27), which implies that $v(x) \leq u(x)$. Interchanging $u$ and $v$, we can also derive $u(x) \leq v(x)$. Therefore, we must have $u \equiv v$. This yields the uniqueness.

This completes the proof of Theorem 4.2 .

\section{The Proof of TheOrem 5}

In this section, we consider a special case where $\Omega$ is an upper half space:

$$
\begin{cases}(-\Delta)_{p}^{s} u(x)=f(u(x)), & x \in \mathbb{R}_{+}^{n}, \\ u(x)>0, & x \in \mathbb{R}_{+}^{n}, \\ u(x)=0, & x \in \mathbb{R}^{n} \backslash \mathbb{R}_{+}^{n} .\end{cases}
$$

We are able to use the sliding method in any direction to obtain a stronger result.

Theorem 5.1. Suppose that $u \in C_{l o c}^{1,1} \cap \mathcal{L}_{s p}$ be a bounded solution of (5.1). Assume that $f$ is continuous and satisfies condition (a)-(c) for some $0<t_{0}<t_{1}<\mu$.

Then $u$ is strictly monotone increasing in $x_{n}$, and moreover it depends on $x_{n}$ only.

Furthermore the bounded solution of (5.1) is unique.

Proof. For $\tau \geq 0$, denote

$$
u_{\tau}(x):=u(x+\tau \nu), \quad w_{\tau}(x):=u(x)-u_{\tau}(x),
$$

where $\nu=\left(\nu_{1}, \nu_{2}, \cdots, \nu_{n}\right)$ with $\nu_{n}>0$.

Similar to the proof of Theorem 4.2, for $\forall \tau>0$, we obtain

$$
w_{\tau}(x)<0, \quad \forall x \in \mathbb{R}^{n},
$$


which implies that $u$ is strictly monotone increasing in any direction $\nu=\left(\nu_{1}, \cdots, \nu_{n}\right)$ with $\nu_{n}>0$.

For each fixed point $x \in \mathbb{R}_{+}^{n}$, let $\nu_{n} \rightarrow 0$. By the continuity of $\nabla u$, we deduce that $\partial_{\nu} u(x) \geq 0$ for any $\nu$ with $\nu_{n}=0$. Replacing $\nu$ by $-\nu$, we obtain $\partial_{\nu} u=0$. Since this is true for all $\nu$ with $\nu_{n}=0$, we conclude that $u$ depends on $x_{n}$ only.

This completes the proof of Theorem 5.1.

\section{Appendix}

In this section, we prove three lemmas.

Lemma 6.1. For $G(t)=|t|^{p-2} t(p \geq 2)$, assume that $t_{1}+t_{2}>0$. Then

$$
G\left(t_{1}+t_{2}\right) \leq 2^{p-2}\left(G\left(t_{1}\right)+G\left(t_{2}\right)\right) .
$$

Proof. Without loss of generality, we may assume that $t_{2}>0$, let $t=\frac{t_{1}}{t_{2}}$. Inequality (6.1) is equivalent to

$$
G(1+t) \leq 2^{p-2}(G(1)+G(t)), t>-1
$$

Let

$$
F(t)=G(1+t)-2^{p-2}(G(1)+G(t))
$$

Assume that $F^{\prime}(t)=0$, we can derive that $t=-\frac{1}{3}$ (local minimum point), $t=1$ (local maximum point), and

We also can calculate

$$
F\left(-\frac{1}{3}\right) \leq 0, \quad F(1)=0
$$

$$
\lim _{t \rightarrow-1^{+}} F(t)=0
$$

It follows that $F(t) \leq 0$ for any $t>-1$.

This completes the proof of the lemma.

Lemma 6.2. Assume that $u$ is the bounded solution of (1.4) and $\Phi \in C_{0}^{\infty}\left(\mathbb{R}^{n}\right)$. For any $0<\delta<1$, then

$$
\left|(-\Delta)_{p}^{s}\left(u+\varepsilon_{k} \Phi\right)(x)-(-\Delta)_{p}^{s} u(x)\right|<\varepsilon_{k} C_{\delta}+C \delta^{p(1-s)} .
$$

Proof. For any $x \in \mathbb{R}^{n}$, we divide the integral into two parts.

$$
\begin{aligned}
(-\Delta)_{p}^{s}\left(u+\varepsilon_{k} \Phi\right)(x)-(-\Delta)_{p}^{s} u(x) \\
=C_{n, s, p} P . V \cdot \int_{\mathbb{R}^{n}} \frac{G\left(u(x)+\varepsilon_{k} \Phi(x)-u(y)-\varepsilon_{k} \Phi(y)\right)-G(u(x)-u(y))}{|x-y|^{n+s p}} d y \\
=C_{n, s, p} P . V \cdot \int_{B_{\delta}(x)} \frac{G\left(u(x)+\varepsilon_{k} \Phi(x)-u(y)-\varepsilon_{k} \Phi(y)\right)-G(u(x)-u(y))}{|x-y|^{n+s p}} d y \\
\quad+C_{n, s, p} \int_{\mathbb{R}^{n} \backslash B_{\delta}(x)} \frac{G\left(u(x)+\varepsilon_{k} \Phi(x)-u(y)-\varepsilon_{k} \Phi(y)\right)-G(u(x)-u(y))}{|x-y|^{n+s p}} d y \\
:=I_{\delta}(x)+J_{\delta}(x) .
\end{aligned}
$$


Now we first estimate $I_{\delta}(x)$. Applying the mean value theorem to the function $G(t)=|t|^{p-2} t$, we derive that there is a constant $C>0$ such that for any two quantities $\mathrm{A}$ and $\mathrm{B}$, it holds

$$
|| A+\left.B\right|^{p-2}(A+B)-|A|^{p-2} A\left|\leq C(|A|+|B|)^{p-2}\right| B \mid .
$$

Define

$$
v(x):=u(x)+\varepsilon_{k} \Phi(x) .
$$

Since $u \in C_{l o c}^{1,1}$ and $\Phi \in C_{0}^{\infty}\left(\mathbb{R}^{n}\right)$, by Taylor expansion, we have

$$
v(x)-v(y)=\nabla v(x) \cdot(x-y)+O\left(|y-x|^{2}\right) .
$$

Let

$$
A=\nabla v(x) \cdot(x-y) \text { and } B=O\left(|y-x|^{2}\right) .
$$

Then it follows from (6.3) that

$$
\begin{aligned}
& || v(x)-\left.v(y)\right|^{p-2}(v(x)-v(y))-|\nabla v(x) \cdot(x-y)|^{p-2} \nabla v(x) \cdot(x-y) \mid \\
& \leq C\left(|\nabla v(x) \cdot(x-y)|+|x-y|^{2}\right)^{p-2}|x-y|^{2} \\
& \leq C\left(|\nabla v(x)|^{p-2}|x-y|^{p} .\right.
\end{aligned}
$$

Then anti-symmetry of $\nabla v(x) \cdot(x-y)$ for $y \in B_{\delta}(x)$ implies

$$
P . V . \int_{B_{\delta}(x)} \frac{|\nabla v(x) \cdot(x-y)|^{p-2} \nabla v(x) \cdot(x-y)}{|x-y|^{n+s p}} d y=0 .
$$

The estimate of the integral of $u$ on $B_{\delta}(x)$ is similar to the estimate of $v$, hence by virtue of (6.4) and (6.5),

$$
\begin{aligned}
\left|I_{\delta}(x)\right| & =C_{n, s, p}\left|P . V . \int_{B_{\delta}(x)} \frac{G\left(u(x)+\varepsilon_{k} \Phi(x)-u(y)-\varepsilon_{k} \Phi(y)\right)-G(u(x)-u(y))}{|x-y|^{n+s p}} d y\right| \\
& \leq C\left(|\nabla v(x)|^{p-2}+|\nabla u(x)|^{p-2}\right) \int_{B_{\delta}(x)} \frac{|x-y|^{p}}{|x-y|^{n+s p}} d y \\
& \leq C \delta^{p(1-s)} .
\end{aligned}
$$

For the estimate of $J_{\delta}$, we have

$$
\begin{aligned}
J_{\delta}(x) & =C_{n, s, p} \int_{\mathbb{R}^{n} \backslash B_{\delta}(x)} \frac{G\left(u(x)+\varepsilon_{k} \Phi(x)-u(y)-\varepsilon_{k} \Phi(y)\right)-G(u(x)-u(y))}{|x-y|^{n+s p}} d y \\
& =\varepsilon_{k} C_{n, s, p} \int_{\mathbb{R}^{n} \backslash B_{\delta}(x)} \frac{(\Phi(x)-\Phi(y)) Q(x, y)}{|x-y|^{n+s p}} d y,
\end{aligned}
$$

where

$$
Q(x, y)=(p-1)(\Phi(x)-\Phi(y)) \int_{0}^{1}\left|u(x)-u(y)+t \varepsilon_{k}(\Phi(x)-\Phi(y))\right|^{p-2} d t,
$$

and we have used the following identity

$$
|b|^{p-2} b-|a|^{p-2} a=(p-1)(b-a) \int_{0}^{1}|a+t(b-a)|^{p-2} d t .
$$

Since $u$ is the bounded solution of (1.4) and $\Phi \in C_{0}^{\infty}\left(\mathbb{R}^{n}\right)$, then

$$
\left|J_{\delta}(x)\right| \leq \frac{C}{\delta^{s p}} \varepsilon_{k}:=C_{\delta} \varepsilon_{k} .
$$


This completes the proof of the lemma.

Lemma 6.3. (A comparison principle) Let $\Gamma$ be a bounded domain in $\mathbb{R}^{n}$. Assume that $u, v \in C_{\text {loc }}^{1,1}(\Gamma) \cap \mathcal{L}_{s p}$ be lower semi-continuous on $\bar{\Gamma}$, and satisfy

$$
\begin{cases}(-\Delta)_{p}^{s} u(x) \geq(-\Delta)_{p}^{s} v(x), & x \in \Gamma, \\ u(x) \geq v(x), & x \in \mathbb{R}^{n} \backslash \Gamma .\end{cases}
$$

Then

$$
u(x) \geq v(x), \quad x \in \Gamma .
$$

If $u(x)=v(x)$ at some point $x \in \Gamma$, then

$$
u(x)=v(x) \text { almost everywhere in } \mathbb{R}^{n} .
$$

Proof. Let

$$
w(x)=u(x)-v(x)
$$

Suppose (6.7) is violated, then since $w$ is lower semi-continuous on $\bar{\Gamma}$, there exists $x^{0}$ in $\Gamma$ such that

$$
w\left(x^{0}\right)=\min _{\Gamma} w(x)<0 .
$$

It follows from the second inequality in (6.6) that

$$
\begin{aligned}
& (-\Delta)_{p}^{s} u\left(x^{0}\right)-(-\Delta)_{p}^{s} v\left(x^{0}\right) \\
& =C_{n, s, p} P . V \cdot \int_{\mathbb{R}^{n}} \frac{G\left(u\left(x^{0}\right)-u(y)\right)-G\left(v\left(x^{0}\right)-v(y)\right)}{\left|x^{0}-y\right|^{n+s p}} d y \\
& \leq C_{n, s, p} \int_{\mathbb{R}^{n} \backslash \Gamma} \frac{G\left(u\left(x^{0}\right)-u(y)\right)-G\left(v\left(x^{0}\right)-v(y)\right)}{\left|x^{0}-y\right|^{n+s p}} d y \\
& <0 .
\end{aligned}
$$

This contradicts the first inequality in (6.6) and hence (6.7) must be true. It follows that if $w\left(x^{0}\right)=0$ at some point $x^{0} \in \Gamma$, then

$$
\begin{aligned}
& (-\Delta)_{p}^{s} u\left(x^{0}\right)-(-\Delta)_{p}^{s} v\left(x^{0}\right) \\
& =C_{n, s, p} P . V \cdot \int_{\mathbb{R}^{n}} \frac{G\left(u\left(x^{0}\right)-u(y)\right)-G\left(v\left(x^{0}\right)-v(y)\right)}{\left|x^{0}-y\right|^{n+s p}} d y \\
& \leq 0,
\end{aligned}
$$

while on the other hand, from the first inequality in (6.6), we should have

$$
(-\Delta)_{p}^{s} u\left(x^{0}\right)-(-\Delta)_{p}^{s} v\left(x^{0}\right) \geq 0
$$

and hence the integral must be 0 . Taking into account that $w$ is already nonnegative, we derive

$$
w(x)=0 \text { almost everywhere in } \mathbb{R}^{n} .
$$

This proves the lemma. 


\section{ACKNOWLEDGEMENTS}

This work was completed while the first author was visiting Yeshiva University. He would like to thank the Department of Mathematical Science at Yeshiva University for the hospitality and the stimulating environment.

\section{REFERENCES}

[1] H. Berestycki, L. Caffarelli and L. Nirenberg, Monotonicity for elliptic equations in an unbounded Lipschitz domain, Comm. Pure Appl. Math., 50 (1997), 1089-1112.

[2] H. Berestycki, L. Caffarelli and L. Nirenberg, Symmetry for elliptic equations in a half space, pp. 27-42 in: Boundary Value Problems for Partial Differential Equations and Applications, RMA Res. Notes Appl. Math., 29, J. L. Lions et al., eds., Masson, Paris, 1993.

[3] H. Berestycki, F. Hamel and R. Monneau, One-dimensional symmetry of bounded entire solutions of some elliptic equations, Duke Math. J., 103 (2000), 375-396.

[4] H. Berestycki and L. Nirenberg, On the method of moving planes and the sliding method, Boletim Soc. Brasil. de Mat. Nova Ser., 22 (1991), 1-37.

[5] I. Birindelli and J. Prajapat, One-dimensional symmetry in the Heisenberg group, Ann. Scuola Norm. Sup. Pisa Cl. Sci., 30 (2001), 269-284.

[6] C. Brandle, E. Colorado, A. de Pablo and U. Sanchez, A concave-convex elliptic problem involving the fractional Laplacian, Proc. Roy. Soc. Edinburgh, 143 (2013) 39-71.

[7] L. Brasco, E. Lindgren and A. Schikorra, Higher Hölder regularity for the fractional p-Laplacian in the superquadratic case, Adv. Math., 338 (2018), 782-846.

[8] L. Caffarelli, B. Gidas and J. Spruck, Asymptotic symmetry and local behavior of semilinear elliptic equations with critical Sobolev growth, Comm. Pure Appl. Math., 42 (1989), 271-297.

[9] L. Caffarelli and L. Silvestre, An extension problem related to the fractional Laplacian, Comm. PDE., 32 (2007), 1245-1260.

[10] L. Caffarelli and L. Silvestre, Regularity theory for fully nonlinear integro-differential equations, Comm. Pure Appl. Math., 62 (2009) 597-638.

[11] S.-Y.A. Chang and P. Yang, On uniqueness of solutions of $n$-th order differential equations in conformal geometry, Math. Res. Lett., 4 (1997), 91-102.

[12] H. Chan and J. Wei, Traveling wave solutions for bistable fractional Allen-Cahn equations with a pyramidal front, J. Differential Equations 262 (2017), no. 9, 4567-4609

[13] W. Chen, Y. Fang and R. Yang, Liouville theorems involving the fractional Laplacian on a half space, Adv. Math., 274 (2015), 167-198.

[14] W. Chen and C. Li, Classification of solutions of some nonlinear elliptic equations, Duke Math. J., 63(3) (1991), 615-622.

[15] W. Chen and C. Li, Methods on Nonlinear Elliptic Equations, AIMS Book Series on Diff. Equa. and Dyn. Sys., Vol. 4, 2010.

[16] W. Chen and C. Li, Maximum principles for the fractional p-Laplacian and symmetry of solutions, Adv. Math., 335 (2018), 735-758.

[17] W. Chen, C. Li and G. Li, Symmetry of solutions for nonlinear problems involving fully nonlinear nonlocal operators, Calc. Var. Partial Differential Equations, 272 (2017) 4131-4157.

[18] W. Chen, C. Li and Y. Li, A direct method of moving planes for fractional Laplacian, Adv. Math., 308 (2017) 404-437.

[19] W. Chen, C. Li and Y. Li, A direct blowing-up and rescaling argument on nonlocal elliptic equations, Internat. J. Math., 27 (2016), 1650064, 20 pp.

[20] W. Chen, C. Li and B. Ou, Classification of solutions for an integral equation, Comm. Pure Appl. Math., 59 (2006), 330-343.

[21] W. Chen, C. Li and B. Ou, Classification of solutions for a system of integral equations, Comm. PDE, 30 (2005), 59-65.

[22] W. Chen, Y. Li and R. Zhang, A direct method of moving spheres on fractional order equations. J. Funct. Anal., 272 (2017), 4131-4157. 
[23] W. Chen and J. Zhu, Indefinite fractional elliptic problem and Liouville theorems, J. Differential Equations, 260 (2016) 4758-4785.

[24] W. Dai, Y. Fang, J. Huang, Y. Qin and B. Wang, Regularity and classification of solutions to static Hartree equations involving fractional Laplacians, Discrete and Continuous Dynamical Systems - A, 39 (2019), no. 3, 1389-1403.

[25] W. Dai, Y. Fang and G. Qin, Classification of positive solutions to fractional order Hartree equations via a direct method of moving planes, J. Differential Equations, 265 (2018), no. 5, 2044-2063.

[26] W. Dai, Z. Liu and G. Lu, Liouville type theorems for PDE and IE systems involving fractional Laplacian on a half space, Potential Analysis, 46 (2017), 569-588.

[27] W. Dai and G. Qin, Classification of nonnegative classical solutions to third-order equations, Adv. Math., 328 (2018), 822-857.

[28] E. De Giorgi, Sulla differenziabilità e l'analiticità delle estremali degli integrali multipli regolari, Mem. Accad. Sci. Torino. Cl. Sci. Fis. Mat. Nat., 3 (1957) 25-43.

[29] E. De Giorgi, "Convergence problems for functionals and operators" in Proceedings of the International Meeting on Recent Methods in Nonlinear Analysis (Rome, 1978), Pitagora, Bologna, 1979, 131-188.

[30] S. Dipierro, N. Soave and E. Valdinoci, On fractional elliptic equations in Lipschitz sets and epigraphs: regularity, monotonicity and rigidity results, Math. Ann. 369 (2017), no. 3-4, 1283-1326.

[31] Z. Du, C. Gui, Y. Sire and J. Wei, Layered solutions for a fractional inhomogeneous Allen-Cahn equation, NoDEA Nonlinear Differential Equations Appl., 23 (2016), no. 3, Art. 29, 26 pp.

[32] Y. Fang and W. Chen, A Liouville type theorem for poly-harmonic Dirichlet problems in a half space, Adv. Math., 229 (2012), 2835-2867.

[33] B. Gidas, W. Ni and L. Nirenberg, Symmetry of positive solutions of nonlinear elliptic equations in $\mathbb{R}^{n}$, Mathematical Analysis and Applications, vol. 7a of the book series Advances in Mathematics, Academic Press, New York, 1981.

[34] B. Gidas, W. Ni and L. Nirenberg, Symmetry and related properties via maximum principle, Comm. Math. Phys., 68 (1979), 209-243.

[35] L. Jin and Y. Li, A Hopf's lemma and the boundary regularity for the fractional p-Laplacian, Discrete Contin. Dyn. Syst., 39 (2019), 1477-1495.

[36] C. Lin, A classification of solutions of a conformally invariant fourth order equation in $\mathbb{R}^{n}$, Comment. Math. Helv., 73 (1998), 206-231.

[37] Z. Liu and W. Dai, A Liouville type theorem for poly-harmonic system with Dirichlet boundary conditions in a half space, Advanced Nonlinear Studies, 15 (2015), 117-134.

[38] Y. Li and M. Zhu, Uniqueness theorems through the method of moving spheres, Duke Math. J., 80 (1995), $383-417$.

[39] Z. Li, Global Hölder regularity of bounded solutions for fractional p-Laplacian, preprint.

[40] Z. Li and Q. Zhang, Subsolutions and Hopf's lemma for fractional p-Laplacian, preprint, arXiv: 1905.00127.

[41] G. Lu and J. Zhu, The axial symmetry and regularity of solutions to an integral equation in a half space, Pacific J. Math., 253 (2011) 455-473.

[42] G. Lu and J. Zhu, Symmetry and regularity of extremals of an integral equation related to the HardySobolev inequality, Calc. Var. Partial Differential Equations, 42 (2011) 563-577.

[43] G. Lu and J. Zhu, An overdetermined problem in Riesz-potential and fractional Laplacian, Nonlinear Anal., 75 (2012) 3036-3048.

[44] L. Ma and L. Zhao, Classification of positive solitary solutions of the nonlinear Choquard equation, Arch. Rational Mech. Anal., 195 (2010), no. 2, 455-467.

[45] O. Savin and E. Valdinoci, $\Gamma$-convergence for nonlocal phase transitions. Ann. Inst. H. Poincaré Anal. NonLinéaire, 29 (2012), 479-500.

[46] L. Silvestre, Regularity of the obstacle problem for a fractional power of the Laplace operator, Comm. Pure Appl. Math., 60 (2007), 67-112.

[47] J. Serrin, A symmetry problem in potential theory, Arch. Rational Mech. Anal., 43 (1971), 304-318.

[48] J. Wei and X. Xu, Classification of solutions of higher order conformally invariant equations, Math. Ann., 313 (1999), no. 2, 207-228. 
[49] R. Zhuo, W. Chen, X. Cui and Z. Yuan, Symmetry and non-existence of solutions for a nonlinear system involving the fractional Laplacian, Disc. Cont. Dyn. Sys., 36 (2016) 1125-1141.

$\dagger$ School of Mathematics and Computer Science, Jiangxi Science and Technology Normal University, Nanchang 330038, P. R. China and Department of Mathematical Sciences, Yeshiva University, New York, NY, USA

E-mail address: liuzhao@mail.bnu.edu.cn

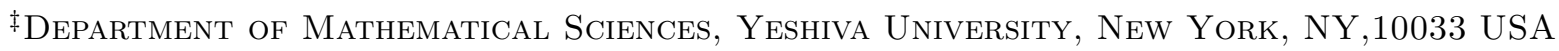

E-mail address: wchen@yu.edu 